\title{
LOS GÉNEROS DE PLANTAS VASCULARES DE LA FLORA DE MÉXICO
}

\author{
JOSÉ LUIS VILLASEÑOR \\ Instituto de Biología, Universidad Nacional Autónoma de México, Departamento de Botánica, \\ Apartado Postal 70-233, C.P. 04510, México, D.F., México \\ Correo-e: vrios@ibiologia.unam.mx
}

\begin{abstract}
Resumen: Un recuento actualizado de la riqueza genérica de plantas vasculares de México incluye 2,804 géneros nativos, distribuidos en 304 familias. Las plantas con flores incluyen el mayor número (2,663), de los cuales 2,117 son dicotiledóneas (Magnoliophyta) y 546 monocotiledóneas (Liliopsida). Se registran además 127 géneros de helechos y plantas afines y 14 de gimnospermas. Se proporciona una lista de nombres, así como el número de especies que registra cada uno de ellos en el país. También se presenta una breve discusión de sus principales formas de crecimiento, de su patrones de riqueza y de su distribución geográfica y ecológica. Se considera que $7.8 \%$ de los géneros (219) son endémicos de México.
\end{abstract}

Palabras clave: diversidad, florística, géneros, México, plantas vasculares.

\begin{abstract}
An updated account of the generic richness of Mexican vascular plants records 2,804 native genera, distributed in 304 families. The flowering plants include the largest number (2,663 genera), 2,117 of them dicotyledons (Magnoliophyta) and 546 monocotyledons (Liliopsida). In addition, 127 genera of ferns and fern allies and 14 of gymnosperms are recorded. A list of generic names is provided, as well as a brief discussion about their richness patterns and their geographical and ecological distribution. Of the total generic richness of Mexico, $7.8 \%$ (219 genera) is considered to be endemic to this country.
\end{abstract}

Key words: diversity, floristics, genera, Mexico, vascular plants.

E género es, dentro de la jerarquía taxonómica, la categoría inmediata más importante por arriba de la especie (Stuessy, 1990). Existen muchas razones para considerar la importancia de un buen conocimiento de los géneros de una región, no nada más de las especies. Por ejemplo, mientras estén los géneros mejor definidos, las especies verdaderamente relacionadas se agruparán como parte de un ensamble monofilético; de esta manera se podrán proponer hipótesis más robustas de relaciones evolutivas y biogeográficas y se facilitarán los estudios florísticos o sistemáticos. McVaugh (1945) propuso una serie de recomendaciones para definir a un género; ejemplos de su aplicación se pueden encontrar en los trabajos de Gillis (1971) o de Grashoff (1975).

Un género es un conjunto de especies caracterizado por varios o muchos caracteres que lo hacen diferente de otro conjunto y que está aislado reproductivamente, es decir, sin evidencia de hibridación (Stuessy, 1990). En otras palabras, un género agrupa una o más especies que tienen más características en común que con cualquier otra especie o con- junto de especies. Esta definición tiene mucho paralelismo con la definición de especie; debido a esto, para varios autores el concepto de género es mucho más difícil de caracterizar, pues si definir una especie es complicado, lo es más agrupar un conjunto de ellas bajo el concepto de género. Sin embargo, desde un punto de vista práctico, el reconocer fácilmente a los géneros ayudará significativamente a identificar a sus miembros, aunque no se sepa a que especie pertenece el ejemplar bajo identificación.

Los géneros, al igual que las demás categorías taxonómicas (desde especies hasta reino) forman parte de la trama que conocemos como biodiversidad. La diversidad total de una región se refiere al número de especies (y toda la jerarquía taxonómica en la que es ubicada dentro de un esquema de clasificación). Mientras más diversidad total encontremos en una región, debemos suponer que allí existe una mayor complejidad ecológica, ya que una mayor biodiversidad sólo podrá coexistir y mantenerse si allí se encuentran mecanismos más complejos, que han evolucionado junto con las especies para regular las interac- 
ciones entre sus miembros. Esta complejidad puede estar manifiesta en una ininteligible conjunción de géneros y familias en las que sus miembros han evolucionado hasta alcanzar la magnitud de la riqueza encontrada. Por estas razones, el entendimiento de la diversidad biológica de una región solamente estará completo cuando se tenga un buen conocimiento de sus especies y de los otros grupos taxonómicos en los que se les ubican.

Reiteradamente se ha dicho que México tiene el privilegio de estar considerado dentro de los países megadiversos del planeta (Mittermeier, 1988; Akeroyd y Synge, 1992; Mittermeier y Goettsch de M., 1992; Heywood y Davis, 1997; Neyra y Durand, 1998; Villaseñor, 2003). Su diversidad poco a poco está siendo conocida, descrita y reportada, aunque a una velocidad desesperadamente lenta, no emparejada con la tasa de destrucción de los ecosistemas donde prospera. Por lo tanto, es importante hacer esfuerzos por sintetizar y analizar la información existente, tanto para dar a conocer lo ya realizado, como para definir estrategias más adecuadas para conocer la riqueza biológica del país en un plazo más adecuado, antes de que sea imposible hacerlo.

En un trabajo previo (Villaseñor, 2003), se hizo un planteamiento de cuál podría ser la riqueza florística conocida de México. En dicho trabajo se presentaron datos para plantas con flores, reportando la riqueza conocida por familia. En este trabajo se presenta un recuento de la diversidad genérica registrada no nada más para las angiospermas, sino para todas las plantas vasculares. El objetivo es proporcionar una lista lo más actualizada posible de los géneros que conforman la riqueza de plantas vasculares en el país, discutir brevemente los patrones de riqueza observados, así como su distribución geográfica y ecológica.

Hasta donde se sabe, solamente hay un trabajo que documenta cuáles son los géneros de plantas con flores de México (Reko, 1946). En dicho trabajo, el Doctor Blas Reko menciona la existencia de 2,189 géneros, distribuidos en 216 familias. Este importante trabajo, que sintetiza el conocimiento florístico de la época, ha sido rebasado en la actualidad tanto en número como en los criterios taxonómicos que definen actualmente a la flora de México. Un repaso por los nombres genéricos citados en el trabajo mencionado revela que más de $20 \%$ de ellos han caído en sinonimia o corresponden a nombres mal aplicados. Por otra parte, tan sólo para angiospermas (plantas con flores), se conocen alrededor de 800 géneros más, lo que representa aproximadamente un $40 \%$ adicional a lo reportado por Reko (1946). En consecuencia, esta contribución pretende ser una actualización de su trabajo, pionero en la documentación de la enorme riqueza florística del país, pero apoyado en los resultados de cinco décadas más de trabajo florístico y taxonómico llevado a cabo por numerosos botánicos, tanto nacionales como extranjeros.

\section{Materiales y métodos}

Como se indicó previamente (Villaseñor, 2003), los datos sobre la diversidad florística de México provienen de una intensa, aunque no exhaustiva, revisión de la literatura florístico-taxonómica, complementada con el estudio continuo del material herborizado y depositado en el Herbario Nacional (MEXU) del Instituto de Biología de la Universidad Nacional Autónoma de México. La literatura revisada incluye desde descripciones originales, listas florísticas, floras o manuales, hasta revisiones taxonómicas y trabajos de índole fitogeográfica. El trabajo se ha visto beneficiado por la gran cantidad de fascículos publicados por los distintos proyectos florísticos que se llevan a cabo en México, como son los de la Flora del Bajío y de Regiones Adyacentes, la Flora de Guerrero, La Flora de Jalisco, la Flora Novo-Galiciana, la Flora de Veracruz, la Flora del Valle de Tehuacán-Cuicatlán, la Etnoflora Yucatanense, etc.

De gran importancia ha sido la consulta de los listados florísticos que para diferentes estados o regiones de México han sido publicados hasta la fecha. De hecho al menos 15 estados ya cuentan con una lista de sus recursos florísticos: Aguascalientes (García et al., 1999); Campeche (Gutiérrez, 2000); Chiapas (Breedlove, 1986); Coahuila (Villarreal-Q., 2001); Durango (González et al., 1991); Hidalgo (Villavicencio-N. et al., 1998); Estado de México (Martínez y Matuda, 1979); Michoacán (Espinosa y Rodríguez-J., 1995, 1996; Rodríguez-J. y Espinosa, 1995, 1996a,b); Morelos (Bonilla-Barbosa y Villaseñor-R., 2003); Querétaro (Argüelles et al., 1991); Quintana Roo (Sousa y Cabrera, 1983); Sinaloa (Vega et al., 1989); Tabasco (Cowan, 1983); Tlaxcala (Acosta et al., 1991) y Veracruz (Sosa y Gómez-Pompa, 1994). Otros trabajos dan cuenta de la diversidad florística de algunos estados adicionales, como son el Distrito Federal (Calderón de R. y Rzedowski, 2001), Baja California y Baja California Sur (Wiggins, 1980) y Yucatán (Durán et al., 2000). Solamente 13 estados no cuentan con un recuento publicado de su riqueza florística (Chihuahua, Colima, Guanajuato, Guerrero, Jalisco, Nayarit, Nuevo León, Oaxaca, Puebla, San Luis Potosí, Sonora, Tamaulipas y Zacatecas).

Diversos catálogos que han sido importantes para sintetizar la información analizada, son los que tratan con la diversidad de grupos específicos en México, como cactáceas (Guzmán et al., 2003), comelináceas (Hunt, 1993), compuestas (Turner y Nesom, 1993; Villaseñor et al., 1998), crasuláceas (Meyrán-G., 2003), cucurbitáceas (Lira et al., 2002), escrofulariáceas (Méndez-L. y Villaseñor-R., 2001), euforbiáceas (Martínez et al., 2002; Steinmann, 2002), gramíneas (Valdés-R. y Dávila-A., 1995), lauráceas (Lorea-Hernández, 2002), monocotiledóneas (Espejo y López-F., 1992, 1993, 1994, 1995, 1996, 1997a,b, 1998, 2000), pinos (Styles, 1993), podostemáceas (Novelo-R. y 
Philbrick, 1997), rubiáceas (Lorence, 1999), teáceas (Luna y Villaseñor, 1996) y otros grupos como malezas (Villaseñor-R. y Espinosa-G., 1998), plantas acuáticas (Lot et al., 1999), plantas introducidas (Villaseñor y EspinosaGarcía, 2004), etc.

Otra fuente importante de información son los inventarios realizados en diversos sitios de México y que cubren extensiones que van desde unas cuantas hectáreas (por ejemplo la reserva ecológica del Pedregal de San Ángel (ValienteBanuet y de Luna-G., 1990) hasta varias decenas de miles de kilómetros cuadrados (por ejemplo el Desierto del Vizcaíno; León de la L. et al., 1995). Hasta la fecha han sido revisados más de 180 inventarios de localidades distribuidas a lo largo y ancho del país, que en conjunto suman más de $300,000 \mathrm{~km}^{2}$ del territorio nacional (J.L. Villaseñor, datos no publicados).

La información contenida en todas esta fuentes ha sido analizada, sintetizada y homogeneizada, para obtener así una lista de la diversidad florística de México. Los datos provenientes de tales referencias representan, como ya se dijo, el trabajo de numerosos botánicos, tanto nacionales como extranjeros y solamente se han evaluado para minimizar los sesgos por sinonimias y malas aplicaciones de nombres y para ubicarlos bajo un contexto taxonómiconomenclatural manejable.

Los nombres genéricos han sido agrupados en categorías taxonómicas superiores siguiendo diferentes propuestas de clasificación. Para el caso de los helechos y plantas afines, los nombres de las familias siguen al criterio adoptado por Mickel y Beitel (1988) y Mickel (1992); para las familias de Gimnospermas se sigue lo aceptado por McVaugh (1992) así como lo adoptado por el Comité Editorial de Flora de Norteamérica (Flora of North America Editorial Comitee, 1993); para las monocotiledóneas (Clase Liliopsida) las familias se agrupan siguiendo la propuesta de Dahlgren et al. (1985) y para las dicotiledóneas la propuesta de Cronquist (1981). Pocas son las adiciones o modificaciones a lo propuesto por estos autores, como por ejemplo la inclusión de la familia Mendonciaceae en Acanthaceae (Daniel, 1992), o la adición de las familias Lacandoniaceae (Martínez y Ramos, 1989), Setchellanthaceae (Iltis, 1999) o Ticodendraceae (GómezLaurito y Gómez-P., 1991).

\section{Resultados}

Las plantas vasculares están representadas en México por 2,804 géneros nativos, de los cuales 127 son helechos y plantas afines, 14 gimnospermas, 546 monocotiledóneas y 2,117 dicotiledóneas (cuadro 1). Estos géneros se incluyen en 304 familias y a su vez contienen un total de 23,424 especies. Estas cifras no incluyen a las plantas introducidas y naturalizadas, que alcanzan una cifra de 618 especies, repartidas en 355 géneros (Villaseñor y Espinosa-García, 2004).
Cuadro 1. Distribución taxonómica de los géneros de plantas vasculares nativos de México.

\begin{tabular}{lrrr}
\hline & Familias & Géneros & Especies \\
\hline Helechos y plantas afines & 49 & 127 & 1,027 \\
Gimnospermas & 7 & 14 & 138 \\
Monocotiledóneas & 49 & 546 & 4,523 \\
Dicotiledóneas & 199 & 2,117 & 17,736 \\
Total & 304 & 2,804 & 23,424 \\
\hline
\end{tabular}

Las dicotiledóneas (Clase Magnoliopsida) constituyen el grupo más diverso de todas las plantas vasculares, representando $75.5 \%$ de la riqueza genérica mexicana. Le siguen en importancia las monocotiledóneas (Clase Liliopsida) y en menor escala los helechos y plantas afines y las gimnospermas. En el cuadro 2 se indican las familias con mayor número de géneros, destacando en los primeros lugares las familias Asteraceae (362 géneros), Poaceae (166), Orchidaceae (157), Fabaceae (92) y Rubiaceae (92). Estas familias destacan igualmente por su número de especies (Villaseñor, 2003).

Cuadro 2. Familias de plantas vasculares de México con mayor número de géneros y géneros con mayor número de especies.

\begin{tabular}{llll}
\hline Familia & Géneros & Género & Especies \\
\hline Asteraceae & 362 & Mammillaria & 306 \\
Poaceae & 166 & Salvia & 292 \\
Orchidaceae & 157 & Euphorbia & 245 \\
Fabaceae & 92 & Dalea & 192 \\
Rubiaceae & 92 & Quercus & 192 \\
Cactaceae & 72 & Tillandsia & 188 \\
Scrophulariaceae & 55 & Verbesina & 179 \\
Malvaceae & 52 & Agave & 173 \\
Acanthaceae & 47 & Solanum & 168 \\
Brassicaceae & 47 & Ipomoea & 162 \\
Euphorbiaceae & 44 & Piper & 153 \\
Apiaceae & 37 & Ageratina & 143 \\
Bignoniaceae & 36 & Mimosa & 138 \\
Apocynaceae & 34 & Muhlenbergia & 130 \\
Mimosaceae & 34 & Croton & 124 \\
Cucurbitaceae & 34 & Carex & 122 \\
Solanaceae & 33 & Opuntia & 120 \\
Lamiaceae & 31 & Stevia & 120 \\
Lamiaceae & 31 & Desmodium & 118 \\
Rosaceae & 30 & Peperomia & 116 \\
& & & \\
\hline & & & \\
& 34 & \\
\hline
\end{tabular}




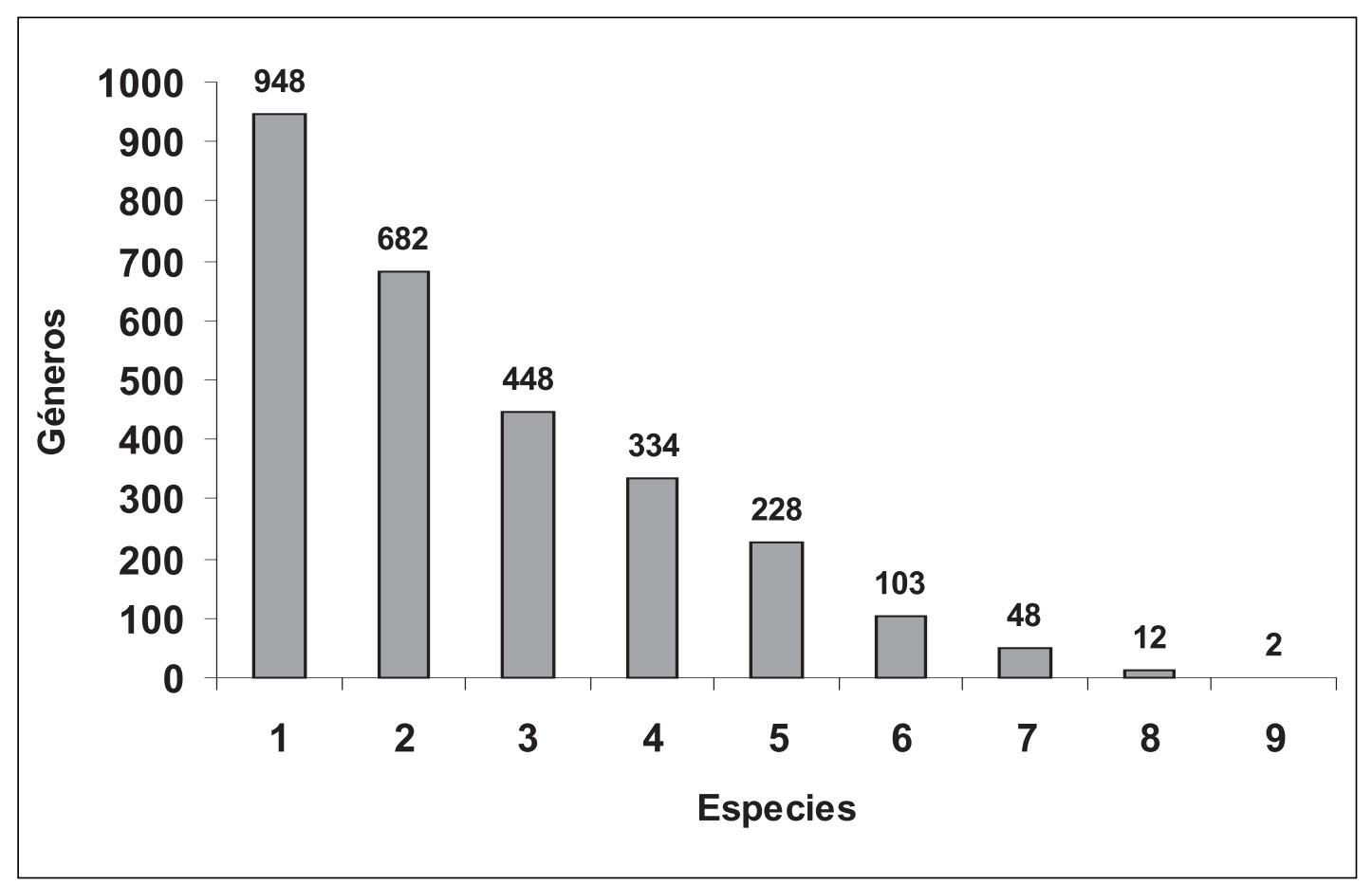

Figura 1. Número de especies por género en la flora vascular de México. Los valores de la abscisa están en escala logarítmica base dos.

En el cuadro 2 se indican también los géneros con mayor número de especies. Entre ellos se encuentran 16 pertenecientes a la Clase Magnoliopsida (dicotiledóneas) y cuatro a la Clase Liliopsida (monocotiledóneas). Tres de esos géneros pertenecen a la familia Asteraceae (Ageratina, Stevia $y$ Verbesina) y cuatro familias contienen a dos géneros, Cactaceae (Mammillaria y Opuntia), Euphorbiaceae (Euphorbia y Croton), Fabaceae (Dalea y Desmodium) y Piperaceae (Piper y Peperomia). Sin embargo, la mayoría de los géneros cuenta con una o pocas especies (figura 1); para 948 géneros solamente se ha registrado una sola especie en el país y 682 cuentan con dos o tres especies, ambos grupos representando $58 \%$ de la diversidad genérica.

La mayoría de los géneros $(1,887$, ó $67 \%)$ incluyen especies con la forma de crecimiento herbácea; le siguen en importancia los arbustos, cuya forma de crecimiento ha sido registrada en especies de al menos 1,187 géneros (42\% de la riqueza genérica). La forma de crecimiento arbórea se registra en 781 géneros (28\%). En el cuadro 3 se indican otras formas de crecimiento importantes y su frecuencia en los géneros mexicanos. Un aspecto interesante es el número de géneros que presentan estrictamente una sola forma de crecimiento; así, por ejemplo, de los 781 géneros a los que se ha documentado la forma de crecimiento arbórea, 166 son estrictamente árboles, es decir, todas sus
Cuadro 3. Formas de crecimiento de los géneros de plantas vasculares mexicanos. Entre paréntesis se indica el número de géneros que estrictamente registra la forma de crecimiento indicada.

\begin{tabular}{lc}
\hline Forma de crecimiento & Géneros \\
\hline Hierbas anuales o perennes & $1,887(1,367)$ \\
Arbustos & $1,187(214)$ \\
Árboles & $781(166)$ \\
Epífitos & 213 \\
Trepadores ${ }^{2}$ & 387 \\
Parásitos & 20 \\
Suculentos & 80 \\
\hline
\end{tabular}

1. Incluyendo sufrútices

2. Incluendo tanto hierbas trepadoras como bejucos

especies son árboles. De igual manera, 18\% (214) de los géneros que registran la forma de crecimiento arbustiva, solamente han sido registrados como arbustos y no como una combinación de formas de crecimiento (por ejemplo árboles y arbustos). Igualmente interesante es encontrar que en alrededor de $72 \%$ de los géneros que registran la forma de crecimiento herbácea, todas sus especies son hierbas (ya sea anuales o perennes, incluyendo sufrútices).

Una evaluación de los patrones de distribución de los géneros por grandes tipos de vegetación (cuadro 4), revela que los bosques templados de México registran el mayor número de géneros $(1,656)$, seguidos por los matorrales 
Cuadro 4. Diversidad genérica en los principales tipos de vegetación de México. Los datos de superficie fueron tomados del Inventario Forestal Nacional (Palacio-Prieto et al., 2000).

\begin{tabular}{lrcc}
\hline Tipos de vegetación & Superficie $\left.\mathbf{( k m}^{2}\right)$ & Géneros & Gen/Logsup \\
\hline Bosques tropicales secos $^{1}$ & 169,264 & 1,276 & 244.0 \\
Bosques tropicales húmedos $^{2}$ & 138,088 & 1,244 & 242.0 \\
Bosques templados $^{3}$ & 328,512 & 1,656 & 300.2 \\
Matorrales xerófilos & 554,539 & 1,381 & 240.4 \\
\hline
\end{tabular}

1. Incluye al bosque espinoso y al bosque tropical caducifolio.

2. Incluye al bosque tropical perennifolio, al bosque tropical subperennifolio y al bosque tropical subcaducifolio.

3. Incluye al bosque mesófilo de montaña, al bosque de Pinus, al bosque de Pinus-Quercus y al bosque de Quercus.

xerófilos $(1,381)$, los bosques tropicales secos $(1,276)$ y los bosques tropicales húmedos $(1,244)$. Los tipos de vegetación con la mayor diversidad también ocupan la mayor superficie (cuadro 4). Un índice de diversidad por tipo de vegetación se puede obtener dividiendo el número de géneros por el $\log _{10}$ de la superficie ocupada por el tipo de vegetación en México. Este índice corresponde más o menos al promedio de géneros por $10 \mathrm{~km}^{2}$ de superficie (Rejmánek, 1996). Al calcular este índice, se observa que excepto por los bosques templados, los otros tipos de vegetación manifiestan una riqueza genérica balanceada, con un promedio de alrededor de 240 géneros por unidad de superficie. Solamente los bosques templados superan este promedio, registrándose un valor de alrededor de 300 géneros en la misma superficie, 60 más que en las otras comunidades.

Rzedowski (1991) y Villaseñor (2003) subrayan que la riqueza específica endémica de México es muy importante, no así la riqueza de géneros endémicos. Rzedowski (1991) consideró que aproximadamente $10 \%$ de los géneros

Cuadro 5. Ejemplos de la distribución geográfica de los géneros de plantas vasculares nativos de México.

\begin{tabular}{lcc}
\hline & Géneros & Porcentaje \\
\hline Endémicos de México & 219 & 7.8 \\
México y Norteamérica & 406 & 14.5 \\
México y Centroamérica & 353 & 12.6 \\
México a Sudamérica & 356 & 12.7 \\
Norteamérica a Centroamérica & 95 & 3.4 \\
Norteamérica a Sudamérica & 574 & 20.5 \\
Presentes en el Viejo Mundo & 305 & 10.9 \\
Pantropicales & 38 & 1.3 \\
Cosmopolitas & 203 & 7.2 \\
\hline
\end{tabular}

restringen su distribución al territorio nacional (alrededor de 240 géneros); Ramamoorthy y Lorence (1987) consideraron que los géneros endémicos superaba la cifra de 300. En este trabajo se reconocen 219 géneros como endémicos del país, una cifra que representa $7.8 \%$ de la riqueza genérica, cifra no muy lejana a la citada por Rzedowski (1991). En la lista de géneros (ver apéndice 1) se indican con un asterisco los géneros reconocidos como endémicos de México.

La mayoría de los géneros son típicamente americanos (cuadro 5). Los géneros que se distribuyen más allá del continente americano representan el 19.4\%; de ellos, 241 $(8.5 \%)$ son de muy amplia distribución, ya sea en los trópicos (pantropicales) o cosmopolitas. Un total de 406 géneros son conocidos solamente de México y el resto de Norteamérica (elementos norteamericanos); por otra parte, 353 géneros se conocen solamente de México y Centroamérica (elementos mesoamericanos). Una cifra no muy distinta de géneros (356) se distribuye de México a Sudamérica (elementos neotropicales). Finalmente, 574 géneros (20.5\%) son de amplia distribución en el continente, registrados desde Norte a Sudamérica (elementos americanos).

En el cuadro 6 se presentan datos preliminares acerca de

Cuadro 6. Riqueza genérica registrada en algunas regiones florísticas o fisiográficas de México. AME = Altiplanicie Mexicana, BAL= Cuenca del Río Balsas, $\mathrm{CAL}=$ Región Mediterránea de Baja California; $\mathrm{CGM}=$ Costa del Golfo de México, $\mathrm{CPA}=$ Costa del Pacífico, $\mathrm{DSON}=$ Desierto Sonorense, $\mathrm{EVT}=$ Eje Volcánico Transversal, $\mathrm{PCNE}=$ Planicie Costera del Noreste de México, $\mathrm{PBCA}=$ Península de Baja California, PYUC= Península de Yucatán, $\mathrm{SMCH}=$ Sierras y Altos de Chiapas, SMOAX $=$ Sierras del Norte de Oaxaca, SMOC = Sierra Madre Occidental, SMOR= Sierra Madre Oriental, $\mathrm{SMS}=$ Sierra Madre del Sur, VTC= Valle de Tehuacán-Cuicatlán, $\mathrm{ZAHQ}=$ Zona Arida de Hidalgo-Querétaro.

\begin{tabular}{lr}
\hline Región & Géneros \\
\hline AME & 1,165 \\
BAL & 1,244 \\
CAL & 527 \\
CGM & 1,437 \\
CPA & 1,381 \\
DSON & 809 \\
EVT & 1,348 \\
PCNE & 775 \\
PBCA & 922 \\
PYUC & 1,281 \\
SMCH & 972 \\
SMOAX & 757 \\
SMOC & 1,016 \\
SMOR & 1,440 \\
SMS & 1,262 \\
VTC & 860 \\
ZAHQ & 586 \\
\hline
\end{tabular}


la riqueza genérica en diferentes regiones florísticas o fisiográficas de México. Los valores de diversidad registrados oscilan desde 527 en la provincia florística de California, correspondiente a la región con clima mediterráneo de México, hasta 1,440 en la Sierra Madre Oriental (promedio $=1,046$; desviación estándar $=288.7$ ). Después de la Sierra Madre Oriental, la región con el mayor número de géneros registrado es la Costa del Golfo de México $(1,437)$, seguida a su vez por el Eje Volcánico Transversal $(1,348)$. Seguramente los valores de riqueza observados están fuertemente correlacionados con el tamaño de la región; las dos regiones con los valores más bajos de riqueza (provincia de California y zona árida hidalguensequeretana), son de los sitios con menor extensión entre las zonas analizadas.

Una evaluación de los patrones de similitud florística entre las regiones analizadas revela una proporción más bien baja de géneros compartidos entre ellas (figura 2). Utilizando el coeficiente de similitud de Jaccard, se observa que solamente la región costera del Golfo tiene un porcentaje de similitud por arriba de $70 \%$ con la península de Yucatán. Todas las demás regiones se agrupan a niveles más bajos de similitud, lo que indica que un alto porcentaje de géneros son más bien de distribución restringida, limitada a una o pocas regiones.

\section{Discusión}

El número de 2,663 géneros de angiospermas (Magnoliophyta) reconocidos en este trabajo incrementa en casi un $20 \%$ la cifra de 2,189 géneros reportada por Reko (1946), y en casi un $10 \%$ la cifra de 2,410 géneros reportada por Rzedowski (1991). El incremento en el número de géneros de helechos y plantas afines (127), con respecto a la cifra reportada por Riba (1993; 110 géneros) es de poco más de $10 \%$. Por un desconocimiento de las cifras para las gimnospermas de México, no es posible comparar los datos aquí proporcionados.

Existe una fuerte correspondencia entre la riqueza de especies y de géneros por familia. Las familias registradas como las más diversas por su número de especies (Villaseñor, 2003), también se registran aquí como las más diversas por su número de géneros. Esta correlación ha sido evaluada para distintas regiones de México (Villaseñor et al., en prensa) y es un patrón característico de la flora vascular de México que puede tener muy importantes repercusiones. Por ejemplo, un mejor conocimiento de la riqueza genérica puede ayudar a que, en plazos más cortos, se tenga un mejor escenario de cuál es la verdadera riqueza florística

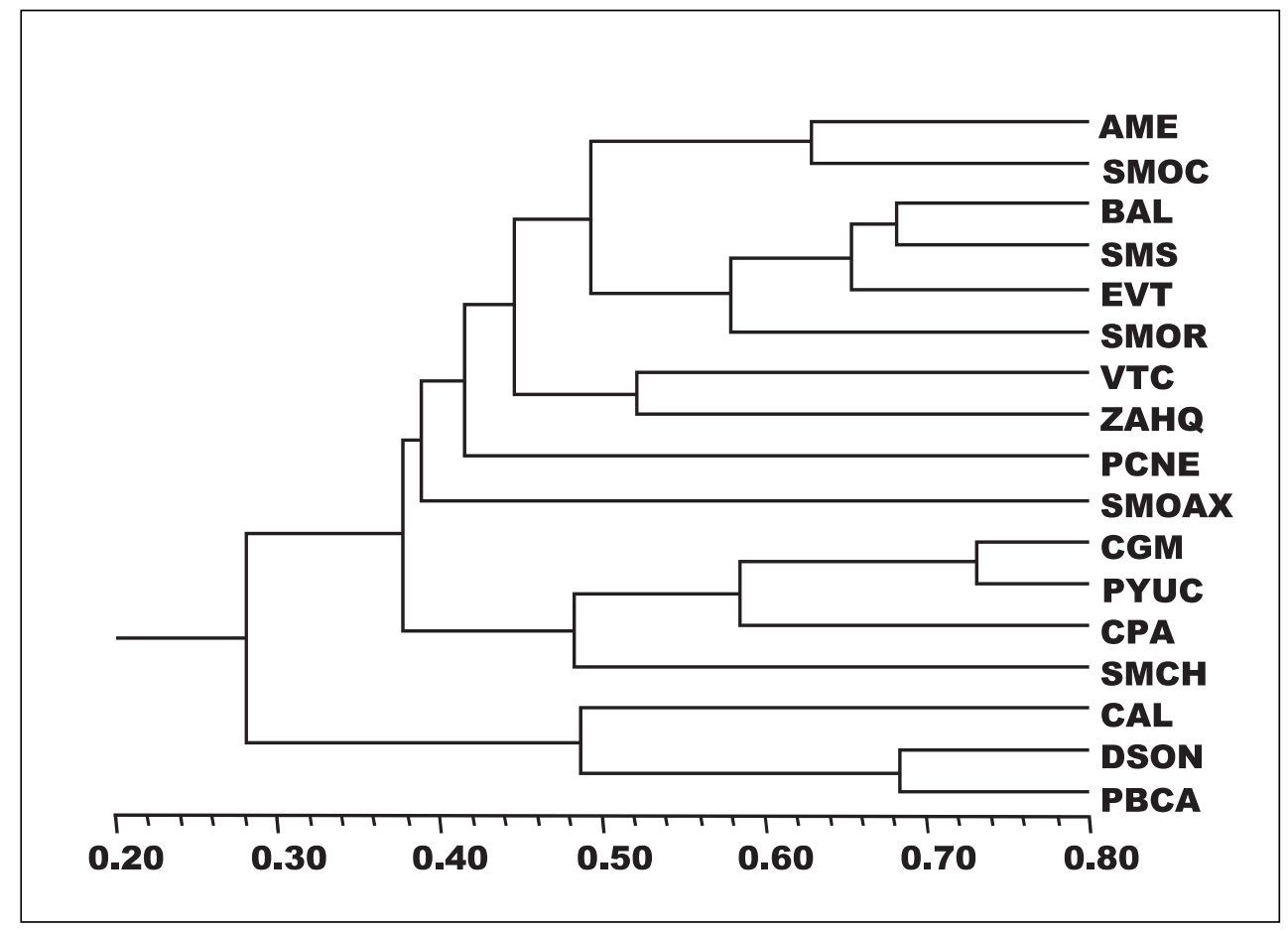

Figura 2. Similitudes genéricas entre diferentes regiones de México. Coeficiente de similitud utilizado: Jaccard; método de agrupamiento: UPGMA (ver el cuadro 6 para el significado de las abreviaturas). 
de México. La importante correlación entre los diferentes taxa de la jerarquía taxonómica (Gaston y Williams, 1993; Andersen, 1995; Balmford et al., 2000; Villaseñor et al., en prensa) ayudará a estimar de mejor manera los patrones de riqueza, sobre todo de países megadiversos como México. Pero lo que es más importante, al evaluar tales patrones se podrán determinar las zonas más importantes para futuros trabajos de inventario, así como para la selección de áreas prioritarias para la conservación de la biodiversidad. Con un buen conocimiento de la riqueza genérica de México, la riqueza de especies y sus patrones de distribución geográfica también será conocida de manera más rápida y eficiente (Villaseñor et al., en prensa).

Las formas de crecimiento tan diversas que se encuentran en la flora de México han llamado mucho la atención de los botánicos (Rzedowski, 1978). Entre los géneros esta diversidad se encuentra ampliamente distribuida, y tal variación puede ayudar a entender en parte el porqué de tanta diversidad de especies. Sin embargo, es importante subrayar que un número importante de géneros (más de 60\%) son conservadores en cuanto a su forma de crecimiento, pues todas sus especies mantienen el mismo tipo. Esta clase de información que se revela al evaluar a la totalidad de la flora de México, puede ayudar en futuras estrategias encaminadas a su mejor conocimiento. Por ejemplo, el saber cuál es la forma de crecimiento de los miembros de un género podrá hacer más eficientes los mecanismos de identificación computarizados, que facilitan y hacen menos tedioso el proceso de asignarle un nombre a los especímenes recolectados en un sitio (ver por ejemplo Murguía y Villaseñor, 1993, 1996).

Los géneros de distribución hacia el norte del continente (elementos norteamericanos) y al norte de Sudamérica (elementos mesoamericanos), refuerzan la idea de que México, junto con Centroamérica y la porción suroeste de Norteamérica, constituyen una región florística de muy alta biodiversidad, donde el nivel de endemismo tanto a nivel genérico como específico se incrementa substancialmente (Myers et al., 2000; Villaseñor, 1990; Rzedowski, 1991). Por otra parte, la distribución de los géneros a lo largo del país (cuadro 6) sugiere que prácticamente todo el territorio nacional presenta una alta riqueza genérica fuertemente correlacionada con una alta riqueza de especies (Villaseñor, 2003; Villaseñor et al., en prensa). Tanto las regiones tropicales del sur de México (como la Costa del Golfo, la Costa del Pacífico, la Cuenca del Balsas o la Península de Yucatán), como las regiones montañosas (por ejemplo la Sierra Madre Oriental, el Eje Volcánico Transversal, la Sierra Madre del Sur o la Sierra Madre Occidental) o las zonas áridas y semiáridas (como la Altiplanicie Mexicana, la Península de Baja California o el Desierto Sonorense), son sitios muy ricos en géneros. Sin embargo, puede afirmarse que no hay un rincón del territorio nacional que no presente un importante número de géneros y especies sil- vestres, excepto quizá ese $24 \%$ del territorio identificado por el inventario forestal nacional (Palacio-Prieto et al., 2000) como superficie completamente transformada por actividades humanas. Pero aun allí es posible encontrar una relevante proporción de malezas y ruderales, tanto nativas como introducidas (Villaseñor-R. y Espinosa-G., 1998; Villaseñor y Espinosa-García, 2004).

Con datos como los aquí mostrados, existe optimismo de que en un futuro muy corto se pueda contar con un inventario más que preliminar de la verdadera riqueza florística de México. Con ello será posible evaluar en su justa medida, el papel que el país juega en la repartición de la biodiversidad a nivel mundial y el papel que debe jugar en su conservación para las futuras generaciones. Ojalá y sirva esta contribución como una motivación para los demás colegas botánicos, a fin de integrarse de manera más entusiasta a la empresa de documentar con datos más claros y específicos, la enorme riqueza florística del país.

\section{Agradecimientos}

Este trabajo representa, como ya se dijo, el esfuerzo de numerosos botánicos, tanto mexicanos como extranjeros, con los que he intercambiado información y experiencias, y quienes me han ayudado a conjuntar gran parte de la información aquí plasmada. De manera particular vaya un agradecimiento a William Anderson, Salvador Arias, Dan Austin, Theodore M. Barkley†, Fernando Chiang, Thomas Daniel, José Delgadillo, Thomas S. Elias, Raquel Galván, Socorro González, Ulises Guzmán, Verónica Juárez, Rafael Lira, Rosalinda Medina, Mario Sousa, Oswaldo Téllez, Susana Valencia, Thomas L. Wendt, y muchos otros que de la memoria se escapan, quienes me han ayudado a resolver muchas dudas y dificultades en el proceso de generación y validación de la información. La hospitalidad y apoyo económico de los doctores Anderson (Michigan), Barkley (Texas) y Elias (Washington) facilitaron en gran medida el arduo trabajo de investigación fuera de casa. Una gran ayuda ha provenido de Enrique Ortiz, quién siempre estuvo atento para resolver las numerosas dudas sobre el manejo de la información en la computadora. Finalmente, un agradecimiento especial a Jorge Meave, quien con su estímulo e interés, hizo que este trabajo llegara a su cul-

\section{Literatura citada}

Acosta P.R., Galindo-F. G.L. y Hernández-C. L.V. 1991. Listado Florístico Preliminar de la Flora Fanerogámica y Micológica del Estado de Tlaxcala. Universidad Autónoma de Tlaxcala, Jardín Botánico Tizatlán, Tlaxcala.

Akeroyd J. y Synge H. 1992. Higher plant diversity. En: Groombridge B. Ed. Global Biodiversity. Status of the Earth's Living Resources, pp. 64-87, Chapman \& Hall, Londres.

Andersen A.N. 1995. Measuring more of biodiversity: Genus richness as a surrogate for species richness in Australian ant 
faunas. Biological Conservation 73:39-43.

Argüelles E., Fernández R. y Zamudio S. 1991. Listado Florístico Preliminar del Estado de Querétaro. Flora del Bajío y de Regiones Adyacentes. Fascículo Complementario II. Instituto de Ecología, A.C., Pátzcuaro.

Balmford A., Lyon A.J.E. y Lang R.M. 2000. Testing the highertaxon approach to conservation planning in a megadiverse group: the macrofungi. Biological Conservation 93:209-217.

Bonilla-Barbosa J.R. y Villaseñor-R. J.L. 2003. Catálogo de la Flora del Estado de Morelos. Centro de Investigaciones Biológicas, Universidad Autónoma del Estado de Morelos, Cuernavaca.

Breedlove D.E. 1986. Flora de Chiapas. Listados Florísticos de México IV. Instituto de Biología, Universidad Nacional Autónoma de México, México, D.F.

Calderón de R. G. y Rzedowski J. 2001. Flora Fanerogámica del Valle de México. 2a ed., Instituto de Ecología, A.C. y Comisión Nacional para el Conocimiento y Uso de la Biodiversidad, Pátzcuaro.

Cowan C.P. 1983. Flora de Tabasco. Listados Florísticos de México I. Instituto de Biología, Universidad Nacional Autónoma de México, México, D.F.

Cronquist A. 1981. An Integrated System of Classification of Flowering Plants. Columbia University Press, Nueva York.

Dahlgren R.M.T., Clifford H.T. y Yeo P.F. 1985. The Families of Monocotyledons. Springer-Verlag, Nueva York.

Daniel T.F. 1992. Acanthaceae: Mendoncioideae of Mexico. Acta Botanica Mexicana 17:53-60.

Durán R., Campos G., Trejo J.C., Simá P., May P.F. y Juan-Q. M. 2000. Listado Florístico de la Península de Yucatán. Centro de Investigación Científica de Yucatán, A.C., Mérida.

Espejo S.A. y López-F. A.R. 1992. Las Monocotiledóneas Mexicanas. Una Sinopsis Florística. Parte I. Consejo Nacional de la Flora de México, A.C. y Universidad Autónoma Metropolitana Iztapalapa, México, D.F.

Espejo S.A. y López-F. A.R. 1993. Las Monocotiledóneas Mexicanas. Una Sinopsis Florística. Parte II. Consejo Nacional de la Flora de México, A.C., Universidad Autónoma Metropolitana Iztapalapa y Comisión Nacional para el Conocimiento y Uso de la Biodiversidad, México, D.F.

Espejo S.A. y López-F. A.R. 1994. Las Monocotiledóneas Mexicanas. Una Sinopsis Florística. Parte III. Consejo Nacional de la Flora de México, A.C., Universidad Autónoma Metropolitana Iztapalapa y Comisión Nacional para el Conocimiento y Uso de la Biodiversidad, México, D.F.

Espejo S.A. y López-F. A.R. 1995. Las Monocotiledóneas Mexicanas. Una Sinopsis Florística. Parte IV. Consejo Nacional de la Flora de México, A.C., Universidad Autónoma Metropolitana Iztapalapa y Comisión Nacional para el Conocimiento y Uso de la Biodiversidad, México, D.F.

Espejo S.A. y López-F. A.R. 1996. Las Monocotiledóneas Mexicanas. Una Sinopsis Florística. Parte VI. Consejo Nacional de la Flora de México, A.C., Universidad Autónoma Metropolitana Iztapalapa y Comisión Nacional para el Conocimiento y Uso de la Biodiversidad, México, D.F.

Espejo S.A. y López-F. A.R. 1997a. Las Monocotiledóneas Mexicanas. Una Sinopsis Florística. Parte V. Consejo Nacional de la Flora de México, A.C., Universidad Autónoma Metropolitana Iztapalapa y Comisión Nacional para el Conocimiento y Uso de la Biodiversidad, México, D.F.
Espejo S.A. y López-F. A.R. 1997b. Las Monocotiledóneas Mexicanas. Una Sinopsis Florística. Parte VII. Consejo Nacional de la Flora de México, A.C., Universidad Autónoma Metropolitana Iztapalapa y Comisión Nacional para el Conocimiento y Uso de la Biodiversidad, México, D.F.

Espejo S.A. y López-F. A.R. 1998. Las Monocotiledóneas Mexicanas. Una Sinopsis Florística. Parte VIII. Consejo Nacional de la Flora de México, A.C., Universidad Autónoma Metropolitana Iztapalapa y Comisión Nacional para el Conocimiento y Uso de la Biodiversidad, México, D.F.

Espejo S.A. y López-F. A.R. 2000. Las Monocotiledóneas Mexicanas. Una Sinopsis Florística. Partes IX a XI. Consejo Nacional de la Flora de México, A.C., Universidad Autónoma Metropolitana Iztapalapa y Comisión Nacional para el Conocimiento y Uso de la Biodiversidad, México, D.F.

Espinosa G.J. y Rodríguez-J. L.S. 1995. Listado Florístico del Estado de Michoacán. Sección II (Angiospermae: Compositae). Flora del Bajío y de Regiones Adyacentes. Fascículo Complementario VII. Instituto de Ecología, A.C., Pátzcuaro.

Espinosa G.J y Rodríguez-J. L.S. 1996. Listado Florístico del Estado de Michoacán. Sección IV (Angiospermae: Fagaceae, Gramineae, Krameriaceae, Leguminosae). Flora del Bajío y de Regiones Adyacentes. Fascículo Complementario XII. Instituto de Ecología, A.C., Pátzcuaro.

Flora of North America Editorial Committe. 1993. Flora of North America North of Mexico. Vol. 2. Pteridophytes and Gymnosperms. Oxford University Press, Nueva York.

Gaston K.J., y Williams P.H. 1993. Mapping the world's species: the higher taxon approach. Biodiversity Letters 1:2-8.

García-R. G., Rosales-C. O., de la Cerda L.M. y Siqueiros-D. M.E. 1999. Listado florístico del estado de Aguascalientes. Scientiae Naturae 1:5-51.

Gillis W.T. 1971. The systematics and ecology of poison-ivy and the poison-oaks (Toxicodendron, Anacardiaceae). Rhodora 73:72-237, 370-540.

Gómez-Laurito J. y Gómez-P. L.D. 1991. Ticodendraceae: a new family of flowering plants. Annals of the Missouri Botanical Garden 78:87-88.

González-E. M., González-E. S.y Herrera-A. Y. 1991. Flora de Durango. Listados Florísticos de México IX. Instituto de Biología, Universidad Nacional Autónoma de México, México, D.F.

Grashoff, J. L. 1975. Metastevia (Compositae: Eupatorieae): a new genus from Mexico. Brittonia 27:69-73.

Gutiérrez-B. C. 2000. Listado Florístico Actualizado del Estado de Campeche. Universidad Autónoma de Campeche, Campeche.

Guzmán U., Arias S. y Dávila P. 2003. Catálogo de Cactáceas Mexicanas. Universidad Nacional Autónoma de México y Comisión Nacional para el Conocimiento y Uso de la Biodiversidad, México, D.F.

Heywood V.H. y Davis S.D.. 1997. Introduction. En: Davis S.D., Heywood V.H., Herrera-MacBryde O., Villa-Lobos J. y Hamilton A.C. Eds. Centres of Plant Diversity. A Guide and Strategy for their Conservation. Volume 3. The Americas, pp. 1-38, The World Wide Fund for Nature (WWF) y UICN-The World Conservation Union, Cambridge.

Hunt D.R. 1993. The Commelinaceae of Mexico. En: Ramamoorthy T.P., Bye R., Lot A. y Fa J. Eds., pp.421-437. Biological Diversity of Mexico. Origins and Distribution. 
Oxford University Press, Nueva York.

Iltis H.H. 1999. Setchellanthaceae (Capparales), a new family for a relictual, glucosinolate-producing endemic of the Mexican deserts. Taxon 48:257-275.

León de la L. J.L., Coria-B. R. del C. y Cansino J. 1995. Reserva de la Biosfera El Vizcaíno, Baja California Sur. Listados Florísticos de México XI. Instituto de Biología, Universidad Nacional Autónoma de México, México, D.F.

Lira R., Villaseñor J.L. y Ortiz E. 2002. A proposal for the conservarion of the family Cucurbitaceae in Mexico. Biodiversity and Conservation 11:1699-1720.

Lorea-Hernández F.G. 2002. La familia Lauraceae en el sur de México: diversidad, distribución y estado de conservación. Boletín de la Sociedad Botánica de México 71:59-70.

Lorence D.H. 1999. A nomenclator of Mexican and Central American Rubiaceae. Monographs in Systematic Botany from the Missouri Botanical Garden Press 73:1-177.

Lot A., Novelo-R. A., Olvera-G. M. y Ramírez-García P. 1999. Catálogo de Angiospermas Acuáticas de México. Cuadernos 33. Instituto de Biología, Universidad Nacional Autónoma de México, México, D.F.

Luna I. y Villaseñor J.L. 1996. Géneros de Theaceae: aspectos taxonómicos y nomenclaturales. Boletín de la Sociedad Botánica de México 59:81-95.

Martínez E. y Ramos C.H. 1989. Lacandoniaceae (Triuridales): una nueva familia de México. Annals of the Missouri Botanical Garden 76:128-135.

Martínez M. y Matuda E. 1979. Flora del Estado de México. 3 Volúmenes. Biblioteca Enciclopédica del Estado de México. México, D.F.

Martínez-G. M., Jiménez-R. J., Cruz-D. R., Juárez-A. E., García R., Cervantes A. y Mejía-H. R. 2002. Los géneros de la familia Euphorbiaceae en México. Anales del Instituto de Biología, UNAM, Serie Botánica 73:155-281.

McVaugh R. 1945. The genus Triodanis Rafinesque, and its relationships to Specularia and Campanula. Wrightia 1:13-52.

McVaugh R. 1992. Gymnosperms and Pteridophytes. Flora Novo-Galiciana Vol. 17. The University of Michigan Herbarium, Ann Arbor, Michigan.

Méndez-L. I. y Villaseñor-R. J.L. 2001. La familia Scrophulariaceae en México: diversidad y distribución. Boletín de la Sociedad Botánica de México 69:89-109.

Meyrán-G. J. 2003. Las Crasuláceas de México. Sociedad Mexicana de Catología, A.C., México, D.F.

Mickel J.T. 1992. Pteridophytes. En: McVaugh R. Ed. Gymnosperms and Pteridophytes. Flora Novo-Galiciana Vol. 17, pp.120-431, The University of Michigan Herbarium, Ann Arbor, Michigan.

Mickel J.T. y Beitel J.M. 1988. Pteridophyte Flora of Oaxaca, Mexico. Memoirs of the New York Botanical Garden 46:1-568.

Mittermeier R.A. 1988. Primate diversity and the tropical forest. En: Wilson E.O. Ed. Biodiversity, pp. 145-154, National Academy Press, Washington, D.C.

Mittermeier R.A. y Goettsch de M. C. 1992. La importancia de la diversidad biológica de México. En: Sarukhán J. y Dirzo R. Comps. México Ante los Retos de la Biodiversidad, pp. 63-73, Comisión Nacional para el Conocimiento y Uso de la Biodiversidad, México, D.F.

Murguía M. y Villaseñor J.L. 1993. FAMEX. Versión 2.0. Clave para familias de plantas con flores (Magnoliophyta) de
México. Programa en Pascal. Asociación de Biólogos Amigos de la Computación, A.C., México, D.F. 56 pp.

Murguía M. y Villaseñor J.L. 1996. GENCOMEX. Versión 1.0. Policlave para la identificación de los géneros de Compositae presentes en México. Programa para Windows. Asociación de Biólogos Amigos de la Computación, A.C. y Comisión Nacional para el Conocimiento y Uso de la Biodiversidad, México, D.F., 36 pp.

Myers N., Mittermeier R.A., da Fonseca G.A.B. y Kent J. 2000. Biodiversity hotspots for conservation priorities. Nature 403:853-858.

Neyra G.L. y Durand S.L. 1998. Biodiversidad. En: Loa L.E. Coord. La Diversidad Biológica de México: Estudio de País, pp. 61-102, Comisión Nacional para el Conocimiento y Uso de la Biodiversidad, México, D.F.

Novelo-R. A. y Philbric C.T. 1997. Taxonomy of Mexican Podostemaceae. Aquatic Botany 57:275-303.

Palacio-Prieto J.L., Bocco G., Velázquez A., Mas J.F., Takaki-T. F., Victoria A., Luna-González L., Gómez-Rodríguez G., López-García J., Palma-M. M., Trejo-Vázquez I., Peralta-H. A., Prado-Molina J., Rodríguez-Aguilar A., Mayorga-Saucedo R. y González-M. F.. 2000. La condición actual de los recursos forestales en México: resultados del Inventario Forestal Nacional 2000. Investigaciones Geográficas, Boletín del Instituto de Geografía, UNAM 43:183-203.

Ramamoorthy T.P. y Lorence D.H. 1987. Species vicariance in the Mexican flora and description of a new species of Salvia (Lamiaceae). Bulletin du Musée National d' Histoire Naturelle, Paris, 4a. ser., sect. B, Adansonia 9:167-175.

Rejmánek M. 1996. Species richness and resistance to invasions. En: Orians G., Dirzo R. y Cushman J.H. Eds. Biodiversity and Ecosystem Processes in Tropical Forests, pp.153-172, Springer-Verlag, Berlín.

Reko B.P. 1946. Los géneros fanerogámicos mexicanos. Boletín de la Sociedad Botánica de México 4:19-40.

Riba R. 1993. Mexican Pteridophytes: distribution and endemism. En: Ramamoorthy T.P., Bye R., Lot A. y Fa J. Eds. Biological Diversity of Mexico. Origins and Distribution, pp. 379-395, Oxford University Press, Nueva York.

Rodríguez-J. L.S. y Espinosa G.J. 1995. Listado Florístico del Estado de Michoacán. Sección I (Gymnospermae; Angiospermae: Acanthaceae-Commelinaceae). Flora del Bajio y de Regiones Adyacentes. Fascículo Complementario VI, Instituto de Ecología, A.C., Pátzcuaro.

Rodríguez-J. L.S. y Espinosa G.J. 1996a. Listado Florístico del Estado de Michoacán. Sección III (Angiospermae: Connaraceae-Myrtaceae excepto Fagaceae, Gramineae, Krameriaceae y Leguminosae). Flora del Bajío y de Regiones Adyacentes. Fascículo Complementario X, Instituto de Ecología, A.C., Pátzcuaro.

Rodríguez-J. L.S. y Espinosa G.J. 1996b. Listado Florístico del Estado de Michoacán. Sección V (Angiospermae: NajadaceaeZygophyllaceae). Flora del Bajío y de Regiones Adyacentes. Fascículo Complementario XV, Instituto de Ecología, A.C., Pátzcuaro, Michoacán.

Rzedowski J. 1978. Vegetación de México. Limusa, México, D.F.

Rzedowski J. 1991. Diversidad y orígenes de la flora fanerogámica de México. Acta Botanica Mexicana 14:3-21.

Sosa V. y Gómez-Pompa A. 1994. Lista Florística. Flora de Veracruz, Fascículo 82, Instituto de Ecología A.C. Xalapa. 
Sousa-S. M. y Cabrera-C. E. 1983. Flora de Quintana Roo. Listados Florísticos de México II. Instituto de Biología, Universidad Nacional Autónoma de México, México, D.F.

Steinmann V.W. 2002. Diversidad y endemismo de la familia Euphorbiaceae en México. Acta Botanica Mexicana 61:61-93.

Stuessy T.F. 1990. Plant Taxonomy. Columbia University Press, Nueva York.

Styles B.T. 1993. Genus Pinus: a Mexican purview. En: Ramamoorthy T.P., Bye R., Lot A. y Fa J. Eds. Biological Diversity of Mexico. Origins and Distribution, pp. 397-420, Oxford University Press, Nueva York.

Turner B.L. y Nesom G.L. 1993. Biogeography, diversity, and endangered or threatened status of Mexican Asteraceae. En: Ramamoorthy T.P., Bye R., Lot A. y Fa J. Eds. Biological Diversity of Mexico. Origins and Distribution, pp. 559-575, Oxford University Press, Nueva York.

Valdés-R. J. y Dávila-A. P.D. 1995. Clasificación de los géneros de Gramíneas (Poaceae) mexicanas. Acta Botanica Mexicana 33:37-50.

Valiente-Banuet A. y de Luna-G. E. 1990. Una lista florística actualizada para la Reserva del Pedregal de San Angel, México, D.F. Acta Botanica Mexicana 9:13-30.

Vega A.R., Bojórquez-B. G.A.y Hernández-A. F. 1989. Flora de Sinaloa. Secretaría de Educación Pública y Universidad Autónoma de Sinaloa, Culiacán.

Fecha de recepción: 4 de octubre de 2004

Versión corregida: 15 de octubre de 2004

Aceptado: 15 de octubre de 2004
Villarreal-Q. J.A. 2001. Flora de Coahuila. Listados Florísticos de México XXIII. Instituto de Biología, Universidad Nacional Autónoma de México, México, D.F.

Villaseñor J.L. 1990. The genera of Asteraceae endemic to Mexico and adjacent regions. Aliso 12:685-692.

Villaseñor J.L. 2003. Diversidad y distribución de las Magnoliophyta de México. Interciencia 28:160-167.

Villaseñor-R. J.L. y Espinosa-G. F.J. 1998. Catálogo de Malezas de México. Universidad Nacional Autónoma de México y Fondo de Cultura Económica. México, D.F.

Villaseñor J.L. y Espinosa-García F. 2004. The alien flowering plants of Mexico. Diversity and Distributions 10:113-123.

Villaseñor J.L., Ibarra G. y Ocaña D. 1998. Strategies for the conservation of Asteraceae in Mexico. Conservation Biology 12:1-10.

Villaseñor J.L., Ibarra-Manríquez G., Meave J.A. y Ortíz E. En prensa. Higher taxa as surrogates of plant biodiversity in a megadiverse country. Conservation Biology.

Villavicencio-N. M.A., Pérez-E. B.E., Ramírez-A. A. 1998. Lista Florística del Estado de Hidalgo. Recopilación Bibliográfica. Universidad Autónoma del Estado de Hidalgo, Centro de Investigaciones Biológicas, Pachuca.

Wiggins I.L. 1980. Flora of Baja California. Stanford University Press, Stanford, California. 
Apéndice 1. Lista de géneros de plantas vasculares de México. Entre paréntesis se indica el número de especies registradas y con un asterisco se indican aquellos géneros endémicos de México.

\section{Helechos y plantas afines}

Familia Adiantaceae

Acrostichum L. (2)

Adiantopsis Fée (2)

Adiantum L. (39)

Ananthocorus Underw. \& Maxon (1)

Anetium Splitg. (1)

Anogramma Link (3)

Antrophyum Kaulf. (6)

Argyrochosma (J.Sm.) Windham (6)

Aspidotis (Nutt. ex Hook.) Copel. (1)

Astrolepis D.M.Benham (2)

Bommeria Fourn. (4)

Cheilanthes Sw. (78)

Cheiloplecton Fée (2)

Cryptogramma R.Br. (1)

Eriosorus Fée (2)

Gymnopteris Bernh. (1)

Hecistopteris J.Sm. (1)

Hemionitis L. (5)

Jamesonia Hook. \& Grev. (1)

Llavea Lag. (1)

Mildella Trevis. (3)

Notholaena R.Br. (22)

Pellaea Link (16)

Pityrogramma Link (7)

Pteris L. (16)

Trismeria Fée (1)

Vittaria Sm. (5)

$\times$ Hemionanthes Mickel (1)

\section{Familia Aspleniaceae}

Adenoderris J.Sm. (1)

Arachniodes Blume (1)

Asplenium L. (75)

Athyrium Roth (8)

Bolbitis Schott (7)

Ctenitis (C.Chr.) Tard. \& C.Chr. (20)

Cyclopeltis J.Sm. (1)

Cystopteris Bernh. (5)

Dictyoxiphium Hook. (1)

Didymochlaena Desv. (1)

Diplazium Sw. (24)

Doryopteris J.Sm. (2)

Dryopteris Adans. (18)

Elaphoglossum Schott ex J.Sm. (59)

Hemidictyum C.Presl (1)

Holodictyum Maxon (1)

Lastreopsis Ching (3)

Lomariopsis Fée (2)

Loxoscaphe T.Moore (1)

Megalastrum J.Sm. (2)
Olfersia Raddi (1)

Onocleopsis Ballard (1)

Peltapteris Link (1)

Phanerophlebia C.Presl (8)

Phyllitis Hill (1)

Polybotrya Humb. \& Bonpl. ex

Willd. (2)

Polystichum Roth (18)

Schaffneria Fee ex T.Moore (1)

Stigmatopteris C.Chr. (3)

Tectaria Cav. (6)

Woodsia R.Br. (5)

\section{Familia Azollaceae}

Azolla Lam. (3)

Familia Blechnaceae

Blechnum L. (15)

Woodwardia Sm. (6)

Familia Cyatheaceae

Alsophila R.Br. (1)

Cnemidaria C.Presl (2)

Cyathea Sm. (13)

Dicksonia L'Her. (1)

Familia Davalliaceae

Nephrolepis Schott (10)

Oleandra Cav. (1)

Familia Dennstaedtiaceae

Dennstaedtia Bernh. (7)

Histiopteris (J.Aghard) J.Sm. (1)

Hypolepis Bernh. (11)

Lindsaea Dryander ex Sm. (6)

Lonchitis L. (1)

Odontosoria Fée (2)

Pteridium Gled. ex Scop. (4)

Saccoloma Kaulf. (2)

Sphenomeris Maxon (1)

Familia Equisetaceae

Equisetum L. (8)

Familia Gleicheniaceae

Dicranopteris Bernh. (2)

Gleichenia Sm. (5)

Familia Grammitidaceae

Ceradenia L.E.Bishop (1)

Cochlidium Kaulf. (2)

Grammitis Sw. (8)

Lellingeria A.R.Sm. \& R.C.Moran (5)
Loxogramme (Blume) C.Presl (1)

Melpomene A.R.Sm. \& R.C.Moran (8)

Micropolypodium Hayata (3)

Terpsichore A.R.Sm. (8)

Familia Hymenophyllaceae

Hymenophyllum Sm. (21)

Trichomanes L. (24)

Familia Isoetaceae

Isoetes L. (6)

Familia Lophosoriaceae

Lophosoria C.Presl (1)

Familia Lycopodiaceae

Huperzia Bernh. (15)

Lycopodiella Holub (1)

Lycopodium L. (9)

Familia Marattiaceae

Danaea Sm. (3)

Marattia Sw. (3)

Familia Marsileaceae

Marsilea L. (6)

Pilularia L. (1)

Familia Metaxyaceae

Metaxya C.Presl (1)

Familia Ophioglossaceae

Botrychium Sw. (6)

Ophioglossum L. (9)

Familia Osmundaceae

Osmunda L. (2)

Familia Parkeriaceae

Ceratopteris Brongn. (2)

Familia Plagiogyriaceae

Plagiogyria (Kunze) Mett. (3)

Familia Polypodiaceae

Campyloneurum C.Presl (9)

Coniogramme Fée (1)

Microgramma C.Presl (6)

Neurodium Fée (1)

Niphidium J.Sm. (1)

Pecluma M.G.Price (8)

Phlebodium (R.Br.) J.Sm. (4)

Pleopeltis Humb. \& Bonpl. ex Willd. (18) 
Pleopodium E.A.Schelpe \&

N.C.Anthony (1)

Polypodium L. (66)

$\times$ Pleopodium E.A.Schelpe \&

N.C.Anthony (1)

Familia Psilotaceae

Psilotum Sw. (2)

Familia Salviniaceae

Salvinia Séguier (2)

Familia Schizaeaceae

Actinostachys Wallich (1)

Anemia Sw. (25)

Lygodium Sw. (3)

Schizaea Sm. (2)

Familia Selaginellaceae

Selaginella Pal. (70)

Familia Thelypteridaceae

Macrothelypteris (H.Ito) Ching (1)

Familia Thelypteridaceae

Thelypteris Schmidel (63)

Familia Thyrsopteridaceae

Cibotium Kaulf. (3)

Culcita C.Presl (1)

\section{Gimnospermas}

Familia Cupressaceae

Calocedrus Kurz (1)

Cupressus L. (5)

Juniperus L. (20)

\section{Familia Cycadaceae}

Dioon Lindl. (11)

Familia Ephedraceae

Ephedra L. (7)

Familia Pinaceae

Abies Mill. (12)

Picea A.Dietr. (3)

Pinus L. (71)

Pseudotsuga Carr. (2)

Familia Podocarpaceae

Podocarpus L'Her. ex Pers. (4)

Familia Taxodiaceae

Taxodium Rich. (1)

Taxus L. (1)
Familia Zamiaceae

Ceratozamia Brongn. (13)

Zamiaceae Zamia L. (10)

III. Angiospermas (Magnoliophyta)

\section{Clase Liliopsida (Monocotiledóneas)}

\section{Familia Agavaceae}

Agave L. (173)

Beschorneria Kunth (9)

Dracaena Vand. ex L. (3)

Furcraea Vent. (16)

Hesperaloe Engelm. (3)

Manfreda Salisb. (26)

Polianthes L. (17)

*Prochnyanthes Schmidel (1)

Yucca L. (37)

\section{Familia Alismataceae}

Alisma L. (1)

Echinodorus Rich. ex Engelm. (11)

Sagittaria L. (12)

\section{Familia Alliaceae}

Allium L. (29)

Behria Greene (1)

* Bessera Schult.f. (3)

* Dandya H.E.Moore (4)

* Jaimehintonia B.L.Turner (1)

Milla Cav. (9)

Muilla S.Watson ex Benth. (1)

Nothoscordum Kunth (3)

* Petronymphe H.E.Moore (1)

\section{Familia Alstroemeriaceae}

Bomarea Mirb. (5)

\section{Familia Amaryllidaceae}

Bloomeria Kellogg (2)

Brodiaea Sm. (2)

Chlidanthus Herb. (1)

Crinum L. (3)

Dichelostemma Kunth (2)

*Diphalangium Schauer (1)

Habranthus Herb. (1)

Hymenocallis Salisb. (30)

Pancratium L. (1)

* Sprekelia Heist. (2)

Triteleiopsis Hoover (1)

Zephyranthes Herbert (36)

\section{Familia Anthericaceae}

Anthericum L. (2)

Echeandia Ortega (53)

\section{Familia Araceae}

Acorus L. (1)

Anthurium Schott (52)

Arisaema Mart. (2)

Dieffenbachia Schott (2)

Dracontium Hill (1)

Monstera Adans. (10)

Philodendron Schott (28)

Pistia L. (1)

Rhodospatha Poepp. (1)

Spathiphyllum Schott (8)

Stenospermation Schott (2)

Syngonium Schott (9)

Xanthosoma Schott (12)

Familia Arecaceae

Acoelorrhaphe Hook.f. (1)

Acrocomia Mart. (1)

Astrocaryum Meyer (1)

Attalea Kunth (2)

Bactris Jacq. (4)

Brahea Mart. (15)

Calyptrogyne H.Wendl. (1)

Chamaedorea Willd. (62)

Coccothrinax Sarg. (1)

Cryosophila Blume (2)

Desmoncus Mart. (5)

Gaussia H.Wendl. (2)

Geonoma Willd. (5)

Pseudophoenix H.Wendl. ex Sarg. (1)

Reinhardtia Liebm. (4)

Roystonea O.F.Cook (2)

Sabal Adans. ex Guers. (8)

Scheelea H.Karst. (2)

Schippia Burret (1)

Synechanthus H.Wendl. (1)

Thrinax L.f. (1)

Washingtonia H.Wendl. (2)

Familia Bromeliaceae

Aechmea Brongn. (4)

Androlepis Brongn. ex Houllet (1)

Billbergia Thunb. (3)

Bromelia L. (6)

Catopsis Griseb. (16)

Chevaliera Gaudich. (1)

Fosterella L.B.Sm. (1)

Greigia Regel (4)

Guzmania Ruiz \& Pav. (2)

Hechtia Klotzsch (48)

Hohenbergiopsis L.B.Sm. \& Read (1)

Pepinia Brongn. ex André (2)

Pitcairnia L'Her. (47)

Platyaechmea (Baker) L.B.Sm. \&

W.J.Kress (2)

Podaechmea (Mez) L.B.Sm. \&

W.J.Kress (3) 
Pothuava Gaudich. (1)

Racinaea M.A.Spencer \& L.B.Sm. (2)

Tillandsia L. (188)

*Ursulaea Read \& Baensch (2)

Viridantha Espejo (6)

Vriesea Lindl. (16)

Familia Burmanniaceae

Apteria Nutt. (1)

Burmannia L. (2)

Dictyostega Miers (1)

Gymnosiphon Blume (3)

Thismia Griff. (1)

Familia Calochortaceae

Calochortus Pursh (22)

Familia Cannaceae

Canna L. (3)

Familia Commelinaceae

Aneilema R.Br. (3)

Callisia Loefl. (18)

Commelina L. (24)

Cymbispatha Pichon (3)

Dichorisandra J.C.Mikan (2)

Gibasis Raf. (18)

*Gibasoides D.R.Hunt (1)

Hadrodemas H.E.Moore (1)

*Matudanthus D.R.Hunt (1)

Phaeosphaerion Hassk. (1)

Rhoeo Hance ex Walp. (1)

*Thyrsanthemum Pichon (4)

Tinantia Scheidw. (10)

Tradescantia L. (56)

Tripogandra Raf. (16)

Weldenia Schult.f. (1)

\section{Familia Convallariaceae}

Maianthemum Weber (6)

Familia Costaceae

Costus L. (10)

Familia Cyclanthaceae

Asplundia Harlin (3)

Carludovica Ruiz \& Pav. (5)

Cyclanthus Poit. (1)

Dicranopygium Harlin (1)

\section{Familia Cymodoceaceae}

Halodule Endl. (2)

Syringodium Kütz. (1)

Familia Cyperaceae

Abildgaardia Vahl (2)
Bulbostylis Kunth (15)

Calyptrocarya Nees (1)

Carex L. (122)

Cladium P.Browne ex Crantz (1)

Cyperus L. (105)

* Cypringlea M.T.Strong (2)

Eleocharis R.Br. (50)

Fimbristylis Vahl (21)

Fuirena Rottb. (6)

Hypolytrum Pers. (2)

* Karinia Reznicek \& McVaugh (1)

Kyllinga Rottb. (3)

Lipocarpha R.Br. (5)

Oxycarium Nees (1)

Pycreus P.Beauv. (3)

Remirea Aubl. (1)

Rhynchospora Vahl (53)

Schoenoplectus (Rchb.) Palla (3)

Schoenus L. (1)

Scirpus L. (15)

Scleria P.J.Bergius (25)

Uncinia Pers. (3)

Familia Dioscoreaceae

Dioscorea L. (70)

*Nanarepenta Matuda (5)

Familia Eriocaulaceae

Eriocaulon L. (10)

Tonina Aubl. (1)

Familia Haemodoraceae

Xiphidium Aubl. (1)

Familia Heliconiaceae

Heliconia L. (23)

Familia Hyacinthaceae

*Hemiphylacus S.Watson (5)

Familia Hydrocharitaceae

Halophila Thouars (2)

Hydromystria G.Mey. (1)

Thalassia Banks \& Sol. ex K.D.

Koenig (1)

Vallisneria L. (1)

Familia Hypoxidaceae

Curculigo Gaertn. (2)

Hypoxis L. (10)

Familia Iridaceae

* Ainea Ravenna (1)

Alophia Herb. (4)

Calydorea Herb. (2)

Cardiostigma Baker (1)
Cipura Aubl. (2)

Cypella Herb. (2)

Eleutherine Herb. (2)

* Fosteria Molseed (1)

Iris L. (1)

Nemastylis Nutt. (5)

Neomarica Sprague (1)

Orthrosanthus Sweet (3)

Rigidella Lindl. (1)

Sessilanthera Molseed \& Cruden (3)

Sisyrinchium Mill. (48)

Tigridia Juss. (40)

Trimezia Salisb. ex Herb. (4)

Familia Juncaceae

Juncus L. (47)

Luzula DC. (7)

Familia Juncaginaceae

Lilaea Humb. \& Bonpl. (1)

Triglochin L. (2)

Familia Lacandoniaceae

* Lacandonia E.Martínez \& Ramos (1)

Familia Lemnaceae

Lemna L. (11)

Spirodela Schleid. (2)

Wolffia Horke ex Schleid. (2)

Wolffiella Hegelm. (3)

Wolffiopsis Harto \& Plas (1)

Familia Liliaceae

Chlorogalum Kunth (1)

Hesperocallis A.Gray (1)

Lilium Hill. (3)

Smilacina Desf. (1)

Triteleia Douglas ex Lindl. (1)

Familia Limnocharitaceae

Hydrocleys Rich. (1)

Limnocharis Humb. \& Bonpl. (2)

Familia Marantaceae

Calathea G.Mey. (15)

Ischnosiphon Körn. (1)

Maranta L. (4)

Stromanthe Son. (2)

Thalia L. (1)

Familia Mayacaceae

Mayaca Aubl. (1)

Familia Melanthiaceae

Schoenocaulon A.Gray (32)

Stenanthium (A.Gray) Kunth (1) 
Veratrum L. (1)

Zigadenus Michx. (8)

\section{Familia Najadaceae}

Najas L. (3)

\section{Familia Nolinaceae}

Beaucarnea Lem. (9)

*Calibanus Rose (2)

Dasylirion Zucc. (24)

Nolina Michx. (21)

\section{Familia Orchidaceae}

Acineta Lindl. (3)

*Alamania Lex. (1)

Amparoa Schltr. (2)

Apatostelis Garay (1)

Arethusa L. (1)

Arpophyllum Lex. (5)

*Artorima Dressler \& G.E.Pollard (1)

Aspidogyne Garay (1)

Barbosella Schltr. (1)

Barkeria Knowles \& Westc. (18)

Beloglottis Schltr. (3)

Bletia Ruiz \& Pav. (22)

Brachystele Schltr. (9)

Brassavola R.Br. (4)

Brassia R.Br. (5)

Bulbophyllum Thouars (6)

Calanthe R.Br. (1)

Campylocentrum Benth. (7)

Catasetum Rich. ex Kunth (3)

Cattleya Lindl. (5)

Caularthron Raf. (1)

Chondrorhyncha Lindl. (2)

Chysis Lindl. (3)

Clowesia Lindl. (5)

Cochleanthes Raf. (1)

Coelia Lindl. (5)

Comparettia Poepp. \& Endl. (1)

Corallorrhiza Gagnebin (13)

Coryanthes Hook. (1)

Corymborkis Thouars (1)

Cranichis Sw. (12)

Cryptarrhena R.Br. (1)

Cuitlauzina La Llave (1)

Cyclopogon C.Presl (7)

Cycnoches Lindl. (3)

Cypripedium L. (3)

Cyrtopodium R.Br. (2)

Deiregyne Schltr. (10)

Dichaea Lindl. (9)

Dichromanthus Garay (1)

*Dignathe Lindl. (1)

Dimerandra Schltr. (1)

Domingoa Schltr. (1)
Dryadella Luer (2)

Elleanthus C.Presl (6)

Encyclia Hook. (82)

Epidendrum L. (105)

Epipactis Zinn (1)

*Erycina Lindl. (2)

Erythrodes Blume (5)

Eulophia R.Br. ex Lindl. (1)

Galeandra Lindl. ex F.A.Bauer (3)

Galeottiella Schltr. (1)

Gongora Ruiz \& Pav. (9)

Goodyera R.Br. (3)

Govenia Lindl. (13)

Gracielanthus R.González \&

Szlach. (2)

Habenaria Willd. (49)

*Hagsatera R.González (2)

Helleriella A.D.Hawkes (2)

Hexadesmia Brongn. (1)

Hexalectris Raf. (8)

Hexisea Lindl. (1)

*Hintonella Ames (1)

Homalopetalum Rolfe (3)

lonopsis Kunth (2)

Isochilus R.Br. (8)

Jacquiniella Schltr. (6)

Kefersteinia Rchb.f. (1)

Kionophyton Garay (2)

Kreodanthus Garay (3)

Lacaena Lindl. (2)

Laelia Lindl. (13)

Lemboglossum Halb. (10)

Leochilus Knowles \& Westc. (8)

Lepanthes Sw. (61)

Lepanthopsis Ames (1)

Leucohyle Klotzsch (1)

Liparis Rich. (11)

Lockhartia Hook. (1)

Lophiaris Raf. (1)

Lycaste Lindl. (8)

Macradenia R.Br. (1)

Macroclinium Barb.Rodr. (3)

Malaxis Sol. ex Sw. (54)

Masdevallia Ruiz \& Pav. (3)

Maxillaria Ruiz \& Pav. (37)

Meiracyllium Rchb.f. (2)

Mendoncella A.D.Hawkes (1)

Mesadenella Pabst \& Garay (1)

Mesoglossum Halb. (1)

*Mexicoa Garay (1)

*Mexipedium V.A.Albert \& M.W. Chase (1)

*Microepidendrum Brieger ex

W.E.Higgins (1)

Mormodes Lindl. 24

Mormolyca Fenzl (1)
Myoxanthus Poepp. \& Endl. (2)

Myrmecophila Rolfe (4)

Nageliella L.O.Williams (1)

* Nezahualcoyotlia R.González (1)

Nidema Britton \& Millsp. (1)

Notylia Lindl. (6)

Oerstedella Rchb.f. (5)

Oncidium Sw. (62)

Ornithocephalus Hook. (6)

Osmoglossum (Schltr.) Schltr. (4)

Pachyphyllum Kunth (1)

Palumbina Rchb.f. (1)

* Papperitzia Rchb.f. (1)

Pelexia Poit. ex Lindl. (8)

Phragmipedium Rolfe (2)

*Physogyne Garay (2)

Piperia Rydb. (1)

Platanthera Rich. (4)

Platystele Schltr. (9)

Platythelys Garay (3)

Pleurothallis R.Br. (83)

Pogonia Juss. (1)

Polystachya Hook. (4)

Ponera Lindl. (7)

Ponthieva R.Br. (12)

Prescottia Lindl. (3)

* Pseudocranichis Garay (1)

Pseudogoodyera Schltr. (2)

Psilochilus Barb.Rodr. (1)

Psygmorchis Dodson \& Dressler (2)

Restrepia Kunth (3)

Restrepiella Garay \& Dunst. (1)

Restrepiopsis Luer (1)

Rhyncholaelia Schltr. (2)

Rhynchostele Rchb. (5)

Rodriguezia Ruiz \& Pav. (1)

Rossioglossum (Schltr.) Garay \&

G.C.Kenn. (4)

Sarcoglottis C.Presl (8)

Scaphosepalum Pfitzer (1)

Scaphyglottis Poepp. \& Endl. (13)

Scelochilus Klotzsch (1)

Schiedeella Schltr. (26)

Schomburgkia Lindl. (3)

Sigmatostalix Rchb.f. (2)

Sobralia Ruiz \& Pav. (8)

Spiranthes Rich. (15)

Stanhopea Frost ex Hook. (16)

Stelis Sw. (21)

Stenorrhynchos Rich. ex Spreng. (3)

*Svenkoeltzia Burns-Bal. (3)

Teuscheria Garay (1)

Trichocentrum Poepp. \& Endl. (2)

Trichopilia Lindl. (2)

Trichosalpinx Luer (7)

Trigonidium Lindl. (1) 
Triphora Nutt. (2)

Tropidia Lindl. (1)

Vanilla Mill. (8)

Warrea Lindl. (1)

Wullschlaegelia Rchb.f. (1)

Xylobium Lindl. (3)

Familia Poaceae

Achnatherum P.Beauv. (8)

Acroceras Stapf (1)

Aegopogon Humb. \& Bonpl. ex Willd. (3)

Agropyron Gaertn. (3)

Agrostis L. (23)

Allolepis Soderstr. \& Decker (1)

Alopecurus L. (3)

Andropogon L. (21)

Anthaenantia P.Beauv. (2)

Anthephora Schreb. (1)

Aristida L. (53)

Arthropogon Nees (1)

Arthrostylidium Rupr. (2)

Arundinella Raddi (4)

Aulonemia Goudot (2)

Axonopus P.Beauv. (12)

Bambusa Schreb. (4)

Bealia Scribn. (1)

Blepharidachne Hackel (1)

Blepharoneuron Nash (2)

Bothriochloa Kuntze (12)

Bouteloua Lag. (58)

Brachiaria (Trin.) Griseb. (7)

Brachypodium P.Beauv. (2)

Briza L. (2)

Bromus L. (21)

Buchloe Engelm. (1)

*Buchlomimus Reeder, C.G. Reeder \& Rzed. (1)

Calamagrostis Adans. (13)

Cathestecum C.Presl (1)

Cenchrus L. (12)

Chaboissaea E.Fourn. (3)

Chaetium Nees (1)

Chaetochloa Scribn. (1)

Chascolytrum Desv. (1)

Chasmanthium Link (2)

Chloris Sw. (12)

Chondrosum Desv. (1)

Chusquea Kunth (24)

Cinna L. (1)

Coelorachis Brongn. (2)

Cottea Kunth (1)

Cryptochloa Swallen (2)

Ctenium Panzer (2)

*Cyclostachya Reeder \& C.G.Reeder (1)
Danthonia DC. (3)

Dasyochloa Rydb. (1)

Deschampsia P.Beauv. (6)

Dichanthelium (Hitchc.

\& Chase) Gould (22)

Diectomis P.Beauv. (2)

Digitaria Heist. ex Scop. (24)

Dissanthelium Trin. (2)

Distichlis Raf. (5)

Echinochloa P.Beauv. (9)

Echinolaena Desv. (2)

Elymus L. (6)

Elyonurus Kunth (2)

Enneapogon Desv. ex P.Beauv. (1)

Enteropogon Nees (2)

Eragrostis Host (44)

Eriochloa Kunth (7)

Eriochrysis P.Beauv. (1)

Erioneuron Nash (2)

Festuca L. (32)

Glyceria R.Br. (4)

Gouinia Fourn. (6)

* Griffithsochloa Pierce (1)

Guadua Kunth (3)

Gymnopogon P.Beauv. (1)

Gynerium Humb. \& Bonpl. (1)

Helleria Nees \& C.Mart. (1)

Heteropogon Pers. (2)

Hierochloe Mathieu (1)

Hilaria Kunth (9)

Homolepis L. (2)

Hordeum L. (4)

Hydrochloa Hartm. (1)

Hymenachne P.Beauv. (2)

Ichnanthus P.Beauv. (8)

Imperata Cirillo (3)

Isachne R.Br. (4)

Ischaemum L. (1)

Ixophorus Schltdl. (1)

Jarava Ruiz \& Pav. (1)

Jouvea Fourn. (2)

Koeleria Pers. (1)

Lasiacis (Griseb.) Hitchc. (18)

Leersia Sw. (5)

Leptochloa P.Beauv. (12)

Leptoloma Chase (1)

Leymus Hochst. (2)

Limnodea L.H.Dewey (1)

Lithachne P.Beauv. (1)

Luziola Juss. ex Gmel. (4)

Lycurus Kunth (2)

Manisuris L. (3)

Matudacalamus Maekawa (1)

Melica L. (6)

Mesosetum Steud. (5)

Metcalfia Conert (1)
Microchloa R.Br. (1)

Monanthochloe Engelm. (1)

Muhlenbergia Schreb. ex Gmel. (130)

Munroa Hack. (1)

Nassella (Trin.) Desv. (8)

Neeragrostis Bush (2)

*Olmeca Soderstr. (2)

Olyra L. (2)

Oplismenus P.Beauv. (6)

Orcuttia Vasey (1)

Orthoclada P.Beauv. (1)

Oryza L. (4)

Oryzopsis Michx. (1)

Otatea (McClure \& Smith)

C.E.Calderón \& Soderstr. (2)

Panicum L. (91)

Pappophorum Schreb. ex Vahl (5)

Paspalidium Stapf (2)

Paspalum L. (89)

Pennisetum Rich. ex Pers. (9)

Pereilema C.Presl (3)

Peyritschia E.Fourn. (2)

Phalaris L. (2)

Pharus P.Browne (4)

Phragmites Trin. (1)

Piptochaetium C.Presl (9)

Pleuraphis Torr. (2)

Poa L. (18)

Polypogon Desf. (2)

Pseudechinolaena Stapf (1)

* Reederochloa Soderstr. \& H.F.Decker (1)

Reimarochloa Hitchc. (1)

Rhipidocladum McClure (4)

Rhytachne Desv. ex Ham. (2)

Sacciolepis Nash (2)

* Schaffnerella Nash (1)

Schizachyrium Nees (19)

Scleropogon Phil. (1)

Setaria P.Beauv. (27)

Setariopsis Scribn. (2)

Sitanion Raf. (3)

* Sohnsia Airy Shaw (1)

Sorghastrum Nash (9)

Sorghum Moench (1)

Spartina Schreb. ex Gmel. (5)

Sphenopholis Scribn. (1)

Sporobolus R.Br. (33)

Steinchisma Raf. (1)

Stipa L. (18)

Streptochaeta Schrad. ex Nees (2)

Streptogyna P.Beauv. (1)

Thrasya Kunth (2)

Trachypogon Nees (8)

Trichloris Fourn. (2)

Tridens Roem. \& Schult. (9) 
Triniochloa Hitchc. (7)

Triplasis P.Beauv. (1)

Tripogon Roem. \& Schult. (1)

Tripsacum L. (12)

Trisetum Pers. (11)

Tristachya Nees (5)

Tuctoria Reeder (1)

Uniola L. (3)

Urochloa P.Beauv. (9)

Zea L. (6)

Zeugites P.Browne (10)

Zizaniopsis Döll \& Asch. ex Döll (1)

\section{Familia Pontederiaceae}

Eichhornia Kunth (3)

Heteranthera Ruiz \& Pav. (9)

Pontederia L. (3)

\section{Familia Potamogetonaceae}

Coleogeton (Rchb.) Les

\& R.R.Haynes (1)

Potamogeton L. (9)

Ruppia L. (2)

Familia Smilacaceae

Smilax L. (28)

Familia Sparganiaceae

Sparganium L. (3)

Familia Triuridaceae

Triuris Miers (1)

Familia Typhaceae

Typha L. (2)

Familia Xyridaceae

Xyris L. (3)

Familia Zannichelliaceae

Zannichellia L. (1)

Familia Zingiberaceae

Renealmia L.f. (6)

Familia Zosteraceae

Phyllospadix Hook. (2)

Zostera L. (1)

\section{Clase Magnoliopsida (Dicotiledóneas)}

\section{Familia Acanthaceae}

Anisacanthus Nees (12)

*Aphanosperma T.F.Daniel (1)

Aphelandra R.Br. (13)

Barleria L. (1)
Beloperone Nees (4)

Blechum P.Browne (2)

Bravaisia DC. (3)

Buceragenia Greenm. (1)

Carlowrightia A.Gray (23)

*Chalarothyrsus Lindau (1)

Chileranthemum Oerst. (3)

Dicliptera Juss. (18)

Dyschoriste Nees (27)

Elytraria Michx. (4)

Glockeria Nees (2)

* Gypsacanthus Lott, Jaramillo

\& Rzed. (1)

Habracanthus Nees (2)

Hansteinia Oerst. (1)

Henrya Nees ex Benth. (4)

*Holographis Nees (15)

*Hoverdenia Nees (1)

Hygrophila R.Br. (1)

*Ixtlania M.E.Jones (1)

Jacobinia Moric. (8)

Justicia L. (89)

Lepidagathis Willd. (1)

Lophostachys Pohl (3)

Louteridium S.Watson (5)

Mendoncia Vell. ex Vand. (3)

* Mexacanthus T.F.Daniel (1)

*Mirandea Rzed. (6)

Nelsonia R.Br. (1)

Odontonema Nees (8)

Poikilacanthus Lindau (3)

Pseuderanthemum Radlk. (9)

Razisea Oerst. (1)

Ruellia L. (62)

Schaueria Nees (1)

Siphonoglossa Oerst. (8)

Spathacanthus Baill. (2)

Staurogyne Wall. (1)

Stenandrium Nees (9)

Stenostephanus Nees (10)

* Tabascina Baill. (1)

Tetramerium Nees (23)

Trybliocalyx Lindau (1)

Yeatesia Small (2)

Familia Aceraceae

Acer L. (5)

Familia Achatocarpaceae

Achatocarpus Triana (3)

Phaulothamnus A.Gray (1)

Familia Actinidiaceae

Saurauia Willd. (27)
Familia Aizoaceae

Sesuvium L. (3)

Trianthema L. (1)

Familia Amaranthaceae

Acanthochiton Torr. (1)

Achyranthes L. (1)

Alternanthera Forssk. (24)

Amaranthus L. (27)

Celosia L. (11)

Chamissoa Kunth (2)

Cyathula Blume (1)

Froelichia Moench (7)

Gomphrena L. (20)

Guilleminea Kunth (2)

Iresine P.Browne (31)

Lagrezia Moq. (1)

Pfaffia Mart. (2)

Philoxerus R.Br. (1)

Pleuropetalum Hook.f. (1)

Tidestromia Standl. (10)

Familia Anacardiaceae

*Actinocheita F.A.Barkley (2)

Astronium Jacq. (2)

*Bonetiella Rzed. (1)

Comocladia L. (5)

Cotinus Mill. (2)

*Cyrtocarpa Kunth (2)

Malosma Nutt. ex Abrams (1)

Mauria Kunth (1)

Metopium P.Browne (1)

Mosquitoxylum Krug \& Urb. (1)

* Pachycormus Coville (3)

Pistacia L. (1)

* Pseudosmodingium Engl. (6)

Rhus L. (41)

Spondias L. (4)

Tapirira Aubl. (3)

Toxicodendron Mill. (7)

Familia Annonaceae

Anaxagorea A.St.-Hil. (1)

Annona L. (16)

Cymbopetalum Benth. (7)

Desmopsis Saff. (8)

Guatteria Ruiz \& Pav. (6)

Malmea R.Fries (1)

Mosannona L.W.Chatrou (1)

Oxandra A.Rich. (3)

Rollinia St.Hil. (2)

Sapranthus Seem. (4)

Stenanona Standl. (1)

*Tridimeris Baill. (2)

Unonopsis R.E.Fries (1)

Xylopia L. (2) 


\section{Familia Apiaceae}

Aletes J.M.Coult. \& Rose (3)

Ammoselinum Torr. \& A.Gray (2)

Angelica L. (1)

Apiastrum Nutt. ex Torr. \& A.Gray (1)

Arracacia Bancr. (28)

Bowlesia Ruiz \& Pav. (2)

Ciclospermum Lag. (1)

Cicuta Mill. (1)

Coaxana J.M.Coult. \& Rose (2)

Conioselinum Hoffm. (1)

*Coulterophytum B.L.Rob. (3)

Cymopterus Raf. (2)

*Dahliaphyllum Constance \& Breedlove (1)

Daucus L. (2)

Donnellsmithia J.M.Coult. \& Rose (20)

Enantiophylla J.M.Coult. \& Rose (1)

Eryngium L. (47)

Harbouria J.M.Coult. \& Rose (1)

Hydrocotyle Crantz (8)

Ligusticum L. (2)

Lilaeopsis Greene (1)

Lomatium Raf. (3)

*Mathiasella Constance \&

C.L.Hitchc. (1)

Micropleura Lag. (1)

Myrrhidendron J.M.Coult. \& Rose (1)

*Neogoezia Hemsl. (3)

Oreomyrrhis Endl. (2)

Osmorhiza Raf. (2)

Ottoa Kunth (1)

Prionosciadium S.Watson (19)

Pseudocymopterus J.M.Coult. \& Rose (1)

Rhodosciadium S.Watson (15)

Sanicula L. (6)

Spananthe Jacq. (1)

Spermolepis Raf. (3)

Tauschia Schltdl. (24)

Yabea Koso-Pol. (1)

\section{Familia Apocynaceae}

Allamanda L. (1)

Alstonia R.Br. (2)

Amsonia Walter (4)

Apocynum L. (4)

Aspidosperma Mart. \& Zucc. (3)

Beaumontia Wall. (1)

Cameraria L. (1)

Cascabela Raf. (3)

Echites Jacq. (9)

Fernaldia Woodson (2)

Forsteronia Meyer (5)

Haplophyton A.DC. (2)

Mandevilla Lindl. (15)
Mesechites Müll.Arg. (1)

Odontadenia Benth. (1)

Pentalinon Voigt (1)

Plocosperma Benth. (1)

Plumeria L. (7)

Prestonia R.Br. (11)

Rauvolfia L. (4)

Rhabdadenia Müll.Arg. (2)

Secondatia DC. (1)

Stemmadenia Benth. (15)

* Streptotrachelus Greenm. (1)

Tabernaemontana L. (4)

Telosiphonia (Woodson) Henr. (7)

Thenardia Kunth (3)

Thevetia Adans. (3)

* Thoreauea J.K.Williams (1)

Tintinnabularia Woodson (2)

Tonduzia Pittier (1)

Trachelospermum Lem. (1)

Urechites Müll. Arg. (1)

Vallesia Ruiz \& Pav. (5)

\section{Familia Aquifoliaceae}

Ilex L. (23)

\section{Familia Araliaceae}

Aralia L. (4)

Dendropanax Decne. \& Planch. (10)

Nothopanax Miq. (1)

Oreopanax Decne. \& Planch. (13)

Schefflera J.R.Forst. \& G.Forst. (1)

Sciadodendron Griseb. (1)

Familia Aristolochiaceae

Aristolochia L. (68)

\section{Familia Asclepiadaceae}

Asclepias L. (81)

Astephanus R.Br. (2)

Blepharodon Decne. (1)

Cynanchum L. (23)

Dictyanthus Decne. (11)

Ditassa R.Br. (1)

Fischeria DC. (1)

Funastrum Fourn. (13)

Gonolobus Michx. (49)

Labidostelma Schltr. (1)

Macroscepis Kunth (5)

Marsdenia R.Br. (27)

Matelea Aubl. (60)

Metalepis Griseb. (1)

Metastelma R.Br. (17)

* Microdactylon Brandegee (1)

Orthosia Decne. (2)

Oxypetalum R.Br. (1)

Pherotrichis Decne. (3)
Polystemma Decne. (1)

Prosthecidiscus Donn.Sm. (1)

Tassadia Decne. (2)

Trichosacme Zucc. (1)

\section{Familia Asteraceae}

Achillea L. (1)

Achyrachaena Schauer (1)

Achyrocline (Less.) DC. (5)

*Achyropappus Kunth (1)

Acmella Rich. ex Pers. (6)

Acourtia D.Don (83)

Adenocaulon Hook. (1)

Adenophyllum Pers. (10)

Adenostemma J.R.Forst. \& G.Forst. (1)

*Adenothamnus D.D.Keck (1)

* Ageratella A.Gray ex S.Watson (3)

Ageratina Spach (143)

Ageratum L. (12)

Agoseris Raf. (1)

Aldama La Llave (2)

Alepidocline S.F.Blake (4)

Alloispermum Willd. (11)

*Alomia Kunth (4)

*Alvordia Brandegee (5)

*Amauria Benth. (3)

Amblyolepis DC. (1)

Amblyopappus Hook. \& Arn. (1)

Ambrosia L. (28)

Amolinia R.M.King \& H.Rob. (1)

Amphiachyris (DC.) Nutt. (1)

Ancistrocarphus A.Gray (1)

Anisocoma Torr. \& A.Gray (1)

Antennaria Gaertn. (1)

Aphanactis Wedd. (1)

Aphanostephus DC. (6)

Archibaccharis Heering (27)

*Arnicastrum Greenm. (3)

Artemisia L. (10)

Aster L. (25)

Astranthium Nutt. (10)

Atrichoseris A.Gray (1)

*Axiniphyllum Benth. (5)

*Aztecaster G.L.Nesom (2)

Baccharis L. (38)

*Baeriopsis J.T.Howell (1)

Bahia Lag. (10)

Baileya Harv. \& A.Gray ex A.Gray (3)

Baltimora L. (2)

Barkleyanthus H.Rob. \& Brettell (1)

Bartlettia A.Gray (1)

Bartlettina R.M.King \& H.Rob. (18)

* Batopilasia G.L.Nesom \& Noyes (1)

Bebbia Greene (3)

Berlandiera DC. (3)

Bidens L. (82) 
Blumea DC. (1)

*Boeberastrum (A.Gray) Rydb. (2)

*Boeberoides (DC.) Strother (1)

*Bolanosa A.Gray (1)

Borrichia Adans. (2)

Bracteantha Anderb. \& Haegi (1)

Brickellia Elliot (88)

Calea L. (10)

Calycadenia DC. (1)

Calycoseris A.Gray (2)

Calyptocarpus Less. (2)

Campuloclinium DC. (1)

Carminatia Moç. ex DC. (3)

Carphochaete A.Gray (7)

Centaurea L. (2)

Chaenactis DC. (12)

Chaetopappa DC. (6)

Chaetymenia Hook. \& Arn. (1)

Chaptalia Vent. (11)

Chloracantha G.L.Nesom, Y.B.Suh,

D.R.Morgan \& B.B.Simpson (4)

Chromolaena DC. (18)

${ }^{*}$ Chromolepis Benth. (1)

Chrysactinia A.Gray (6)

Chrysanthellum Rich. (10)

Chrysothamnus Nutt. (2)

Cirsium L. (48)

Clappia A.Gray (1)

Clibadium L. (1)

Comaclinium Scheidw. \& Planch. (1)

Conoclinium DC. (3)

Conyza Less. (6)

Coreocarpus Benth. (12)

Coreopsis L. (29)

Cosmos Cav. (42)

Cotula L. (1)

*Coulterella Vasey \& Rose (1)

Crepis L. (1)

Critonia P.Browne (16)

Critoniadelphus R.M.King \& H.Rob. (1)

Critoniopsis Sch.Bip. (21)

Cymophora B.L.Rob. (4)

Cyrtocymura H.Rob. (1)

Dahlia Cav. (36)

*Damnxanthodium Strother (1)

Decachaeta DC. (7)

Delilia Spreng. (1)

Desmanthodium Benth. (5)

Dichaetophora A.Gray (1)

Dicoria Torr. \& A.Gray (3)

Dicranocarpus A.Gray (1)

* Digitacalia Pippen (6)

*Dugesia A.Gray (1)

*Dyscritothamnus B.L.Rob. (2)

Dyssodia Cav. (5)

Eclipta L. (1)
Egletes Cass. (2)

Elephantopus L. (3)

Eleutheranthera Poit. ex Bosc (1)

Encelia Adans. (15)

Engelmannia Torr. \& A.Gray

ex Nutt. (1)

Enydra Lour. (1)

Epaltes Cass. (1)

Erechtites Raf. (2)

Ericameria Nutt. (16)

Erigeron L. (89)

Eriophyllum Lag. (7)

*Eryngiophyllum Greenm. (2)

Espejoa DC. (1)

Eupatoriastrum Greenm. (5)

*Euphrosyne DC. (1)

*Eutetras A.Gray (2)

Euthamia (Nutt.) Nutt. (1)

*Faxonia Brandegee (1)

Filago L. (4)

Flaveria Juss. (16)

Fleischmannia Sch.Bip. (22)

Fleischmanniopsis R.M.King

\& H.Rob. (2)

Florestina Cass. (8)

Flourensia DC. (13)

Flyriella R.M.King \& H.Rob. (4)

Gaillardia Foug. (18)

Galeana La Llave (1)

Galinsoga Ruiz \& Pav. (10)

Gamochaeta Wedd. (6)

*Geissolepis B.L.Rob. (1)

Geraea Torr. \& A.Gray (2)

Gnaphaliothamnus Kirp. (9)

Gnaphalium L. (11)

Gochnatia Kunth (6)

Goldmanella Greenm. (1)

* Greenmaniella W.M.Sharp (1)

Grindelia Willd. (29)

Guardiola Cerv. ex Humb.

\& Bonpl. (12)

Gutierrezia Lag. (15)

Gymnocoronis DC. (1)

* Gymnolaena (DC.) Rydb. (3)

Gymnosperma Less. (1)

Haploesthes A.Gray (5)

Harleya S.F.Blake (1)

Hazardia Greene (10)

Hebeclinium DC. (1)

Hedosyne Strother (1)

Helenium L. (14)

Helianthella Torr. \& A.Gray (6)

Helianthus L. (13)

Heliomeris Nutt. (6)

Heliopsis Pers. (10)

Hemizonia DC. (14)
*Henricksonia B.L.Turner (1)

Heterosperma Cav. (3)

Heterotheca Cass. (21)

Hidalgoa La Llave (4)

Hieracium L. (19)

*Hofmeisteria Walp. (12)

Holocarpha (DC.) Greene (1)

Hulsea Torr. \& A.Gray (3)

*Hybridella Cass. (2)

*Hydropectis Rydb. (3)

Hymenoclea Torr. \& A.Gray (4)

Hymenopappus L'Her. (7)

Hymenothrix A.Gray (5)

Hymenoxys Cass. (16)

* Iostephane Benth. (4)

Isocarpha R.Br. (2)

Isocoma Nutt. (18)

Iva L. (5)

Jaegeria Kunth (10)

*Jaliscoa S.Watson (3)

Jaumea Pers. (1)

Jefea Strother (4)

Jungia L.f. (2)

Koanophyllon Arruda (21)

Krigia Schreb. (1)

Kyrsteniopsis R.M.King \& H.Rob. (8)

Lactuca L. (7)

Laennecia Cass. (12)

Lagascea Cav. (11)

Lasianthaea DC. (26)

Lasthenia Cass. (3)

Layia Hook. \& Arn. ex DC. (2)

Leibnitzia Cass. (2)

*Leiboldia Schltdl. ex Gleason (2)

Lepidaploa (Cass.) Cass. (8)

Lepidonia S.F.Blake (5)

Lepidospartum (A.Gray) A.Gray (1)

Lessingia Cham. (4)

*Leucactinia Rydb. (1)

Liabum Adans. (1)

Liatris Gaertn. ex Schreb. (1)

Lindheimera A.Gray \& Engelm. (1)

*Loxothysanus B.L.Rob. (2)

Lundellianthus H.Rob. (4)

Lygodesmia D.Don (2)

Machaeranthera Nees (32)

Macvaughiella R.M.King \& H.Rob. (2)

Madia Molina (5)

Malacothrix DC. (12)

Malperia S.Watson (1)

*Marshalljohnstonia Henr. (1)

Melampodium L. (38)

Melanthera Rohr (2)

*Mexerion G.L.Nesom (2)

*Mexianthus B.L.Rob. (1)

Micropus L. (1) 
Microseris D.Don (4)

Microspermum Lag. (9)

Mikania Willd. (16)

Milleria L. (2)

Monoptilon Torr. \& A.Gray (2)

Montanoa Cerv. (23)

Nelsonianthus H.Rob. \& Brettell (2)

Neomirandea R.M.King \& H.Rob. (2)

*Nesomia B.L.Turner (1)

Neurolaena R.Br. (10)

Nicolletia A.Gray (3)

*Olivaea Sch.Bip. ex Benth. (2)

Onoseris Willd. (1)

Oreochrysum Rydb. (1)

Oritrophium (Kunth) Cuatrec. (2)

Orthopappus Gleason (1)

Osbertia Greene (2)

Oteiza La Llave (3)

Otopappus Benth. (13)

Oxylobus (Moç. ex DC.) A.Gray (5)

*Oxypappus Benth. (1)

Packera A.Löve \& D.Löve (17)

Palafoxia Lag. (11)

Parthenice A.Gray (2)

Parthenium L. (16)

Pectis L. (59)

* Pelucha S.Watson (1)

Pentacalia Cass. (4)

Pentachaeta Nutt. (1)

Pericome A.Gray (2)

Perityle Benth. (48)

Perymenium Schrad. (51)

Peteravenia R.M.King \& H.Rob. (3)

Peucephyllum A.Gray (1)

Philactis Schrad. (4)

Pinaropappus Less. (13)

* Pippenalia McVaugh (1)

Piptocarpha R.Br. (1)

Piqueria Cav. (6)

Pittocaulon H.Rob. \& Brettell (6)

Pityopsis Nutt. (1)

*Plagiolophus Greenm. (1)

Plateilema (A.Gray) Cockerell (1)

Pleurocoronis R.M.King \& H.Rob. (3)

Pluchea Cass. (8)

Podachaenium Benth. (3)

Porophyllum Adans. (19)

Prenanthella Rydb. (1)

Psacaliopsis H.Rob. \& Brettell (4)

Psacalium Cass. (48)

Psathyrotes (Nutt.) A.Gray (1)

Psathyrotopsis Rydb. (3)

Pseudelephantopus Rohr (1)

Pseudoclappia Rydb. (1)

Pseudognaphalium Kirp. (32)

Pseudogynoxys (Greenm.) Cabrera (3)
Psilactis A.Gray (5)

Psilocarphus Nutt. (3)

Psilostrophe DC. (6)

Pterocaulon Elliot (1)

Pyrrhopappus DC. (1)

Rafinesquia Nutt. (2)

Ratibida Raf. (7)

Rayjacksonia R.L.Hartm.

\& M.A.Lane (1)

Rensonia S.F.Blake (1)

Robinsonecio T.M.Barkley

\& J.P.Janovec (2)

Rojasianthe Standl. \& Steyerm. (1)

Roldana La Llave (57)

Rudbeckia L. (1)

Rumfordia DC. (10)

Sabazia Cass. (12)

Salmea DC. (4)

Sanvitalia Lam. (5)

Sartwellia A.Gray (4)

Schistocarpha Less. (7)

Schkuhria Roth (3)

Sclerocarpus Jacq. (9)

Selloa Kunth (1)

Senecio L. (73)

Seriphidium (Besser ex Hook.)

Fourr. (2)

Sigesbeckia L. (3)

Simsia Pers. (16)

Sinclairia Hook. \& Arn. (27)

Smallanthus Mack. (8)

Solidago L. (21)

Sphaeromeria Nutt. (1)

Spilanthes Jacq. (1)

Spiracantha Kunth (1)

Squamopappus R.K.Jansen,

N.A.Harriman \& Urbatsch (1)

Stachycephalum Sch.Bip. ex Benth. (1)

* Stenocarpha S.F.Blake (2)

Stenocephalum Sch.Bip. (1)

Stenotus Nutt. (1)

* Stephanodoria Greene (1)

Stephanomeria Nutt. (11)

Stevia Cav. (120)

Steviopsis R.M.King \& H.Rob. (10)

*Stramentopappus H.Rob.

\& V.A.Funk (1)

* Strotheria B.L.Turner (1)

Struchium P.Browne (1)

* Stuessya B.L.Turner \& F.G.Davies (3)

Stylocline Nutt. (3)

Synedrella Gaertn. (1)

Tagetes L. (29)

Tamaulipa R.M.King \& H.Rob. (1)

Taraxacum Weber (3)

*Tehuana Panero \& Villaseñor (1)
Telanthophora H.Rob. \& Brettell (8)

Tetrachyron Schltr. (8)

Tetradymia DC. (1)

Tetragonotheca L. (1)

Thelechitonia Cuatrec. (1)

Thelesperma Less. (14)

Thymophylla Lag. (15)

Tithonia Desf. (12)

*Tomentaurum G.L.Nesom (1)

Townsendia Hook. (3)

Trichocoronis A.Gray (4)

*Trichocoryne S.F.Blake (1)

Trichoptilium A.Gray (1)

Trichospira Kunth (1)

Tridax L. (26)

Trigonospermum Less. (5)

Trixis P.Browne (23)

Tuxtla Villaseñor \& Strother (1)

*Urbinella Greenm. (1)

Varilla A.Gray (3)

Venegasia DC. (1)

Verbesina L. (179)

Vernonanthura H.Rob. (8)

Vernonia Schreb. (15)

* Vigethia W.A.Weber (1)

Viguiera Kunth (102)

Villanova Lag. (1)

* Villasenoria B.L.Clark (1)

Wamalchitamia Strother (4)

Wedelia Jacq. (26)

Werneria Kunth (1)

Wyethia Nutt. (1)

Xanthium L. (2)

Xanthocephalum Willd. (6)

Xylorhiza Nutt. (2)

Xylothamia G.L.Nesom, Y.B.Suh,

D.R.Morgan \& B.B.Simpson (9)

* Zaluzania Pers. (11)

*Zandera D.L.Schulz (3)

Zexmenia La Llave (2)

Zinnia L. (25)

Zyzyxia Strother (1)

Familia Balanophoraceae

Helosis Rich. (2)

Langsdorffia C.Agardh (1)

Familia Balsaminaceae

Impatiens L. (3)

Familia Basellaceae

Anredera Juss. (3)

Familia Bataceae

Batis L. (1) 
Familia Begoniaceae

Begonia L. (92)

Familia Berberidaceae

Berberis L. (35)

Familia Betulaceae

Alnus Hill (9)

Carpinus L. (2)

Ostrya Scop. (1)

\section{Familia Bignoniaceae}

Adenocalymma Benth. (5)

Amphilophium Kunth (2)

Amphitecna Miers (12)

Anemopaegma C.Mart. ex Meisn. (3)

Arrabidaea DC. (14)

Astianthus Don (1)

Callichlamys Miq. (1)

Campsis Lour. (1)

Ceratophytum Pittier (1)

Chilopsis Don (3)

Chodanthus Hassl. (1)

Clytostoma Miers ex Bureau (1)

Crescentia L. (2)

Cybistax C.Mart. ex Meisn. (1)

Cydista Miers (5)

Distictis C.Mart. ex Meisn. (2)

Godmania Hemsl. (1)

Lundia DC. (1)

Macfadyena A.DC. (3)

Mansoa DC. (3)

Martinella Baill. (1)

Melloa Bureau (1)

Mussatia Bureau ex Baill. (1)

Pachyptera DC. ex Meisn. (1)

Paragonia Bureau (1)

Parmentiera DC. (3)

Pithecoctenium C.Mart. ex Meisn. (1)

Pseudocatalpa A.H.Gentry (1)

Saritaea Dugand (1)

Schlegelia Miq. (2)

Stizophyllum Miers (1)

Tabebuia Gomes (7)

Tecoma Juss. (1)

Tourretia Foug. (1)

Tynanthus Miers (1)

Xylophragma Sprague (1)

\section{Familia Bixaceae}

Amoreuxia Moç. \& Sessé ex DC. (4)

Bixa L. (3)

Cochlospermum Kunth (1)

Familia Bombacaceae

Bernoullia Oliver (1)
Ceiba Medik. (5)

Ochroma Sw. (1)

Pachira Aubl. (2)

Pseudobombax Dugand (2)

Quararibea Aubl. (7)

\section{Familia Boraginaceae}

Allocarya Greene (3)

Amsinckia Spach (4)

Antiphytum DC. ex Meisn. (7)

Antrophora I.M.Johnst. (1)

Bourreria P.Browne (17)

Cordia L. (56)

Cryptantha Lehm. ex G.Don (40)

Cynoglossum L. (3)

Ehretia L. (3)

Eremocarya Greene (2)

Euploca Nutt. (1)

Hackelia Opiz (6)

Harpagonella A.Gray (1)

Heliotropium L. (31)

Lappula Moench (3)

*Lasiarrhenum I.M.Johnst. (3)

Lithospermum L. (30)

Macromeria D.Don (10)

*Mimophytum Greenm. (1)

Omphalodes Mill. (5)

Onosmodium Walpers (4)

Pectocarya DC. ex Meisn. (7)

Plagiobothrys Fisch. \& C.A.Mey. (7)

Rochefortia Sw. (2)

Tiquilia Pers. (11)

Tournefortia L. (22)

\section{Familia Brassicaceae}

Arabis L. (6)

* Asta Klotzsch ex O.E.Schulz (2)

Athysanus Greene (1)

Aurinia Desv. (1)

Cakile Mill. (6)

Capsella Medik. (2)

Cardamine L. (7)

Caulanthus S.Watson (5)

*Cibotarium O.E.Schulz (3)

Coelophragmus O.E.Schulz (1)

Dentaria L. (1)

Descurainia Webb \& Berthel (11)

Dithyrea Harv. (4)

Draba L. (10)

Dryopetalon A.Gray (6)

Erysimum L. (8)

Guillenia Greene (1)

Halimolobos Wittst. (7)

Heterodraba Greene (1)

lodanthus Torr. \& A.Gray (2)

Lepidium L. (24)
Lesquerella S.Watson (19)

Lyrocarpa Hook. \& Harv. ex Harv. (4)

Mancoa Wedd. (6)

Nasturtium Adans. (1)

Nerisyrenia Greene (8)

* Ornithocarpa Rose (1)

Pennellia Nieuwl. (8)

* Rhaphanorhyncha Rollins (1)

Rollinsia Al-Shehbaz (1)

Romanschulzia O.E.Schulz (3)

Rorippa Scop. (10)

Schoenocrambre Greene (1)

* Scoliaxon Payson (1)

Selenia Nutt. (2)

Sibara Greene (5)

Sisymbrium L. (3)

* Sphaerocardamum Schauer (2)

Stanleya Nutt. (1)

Streptanthella Rydb. (1)

Streptanthus Nutt. (6)

Synthlipsis A.Gray (3)

Thelypodiopsis Rydb. (6)

Thelypodium Endl. (3)

Thlaspi L. (2)

Thysanocarpus Hook. (5)

Tropidocarpum Hook. (1)

Familia Brunelliaceae

Brunellia Ruiz \& Pav. (3)

Familia Buddlejaceae

Buddleja L. (21)

Emorya Torr. (2)

\section{Familia Burseraceae}

* Beiselia Forman (1)

Bursera Jacq. (95)

Commiphora Jacq. (1)

Protium Burm.f. (3)

\section{Familia Buxaceae}

Buxus L. (6)

Sarcococca Lindl. (1)

Familia Cabombaceae

Brasenia Schreb. (1)

Cabomba Aubl. (3)

Familia Cactaceae

Acanthocereus Britton \& Rose (6)

*Acharagma (N.P.Taylor \&

A.D.Zimmerman) Glass (2)

Ancistrocactus Britton \& Rose (2)

Anisocereus Backeb. (1)

*Aporocactus Lem. (2)

Ariocarpus Scheidw. (10) 
Astrophytum Lem. (4)

*Aztekium Boed. (2)

* Backebergia Bravo (1)

Bergerocactus Britton \& Rose (1)

Carnegiea Britton \& Rose (1)

*Cephalocereus Pfeiff. (5)

*Cochemiea Walton (5)

Coryphantha Lem. (56)

*Cumarinia Buxb. (1)

Cylindropuntia (Engelm.) F.M.

Knuth (44)

*Digitostigma Velazco \& Nevárez (1)

Disocactus Lindl. (6)

Echinocactus Link \& Otto (7)

Echinocereus Engelm. (102)

Echinomastus Britton \& Rose (11)

Epiphyllum Haw. (9)

Epithelantha Weber ex Britton

\& Rose (4)

Escobaria Britton \& Rose (15)

*Escontria Rose (1)

Ferocactus Britton \& Rose (37)

* Geohintonia Glass \& F.Maurice (1)

Glandulicactus Backeb. (3)

Grusonia F.Rchb. \& K.Schum. (14)

*Hamatocactus Britton \& Rose (3)

Heliocereus Britton \& Rose (5)

Hylocereus Britton \& Rose (3)

*Leuchtenbergia Hook. (1)

Lophocereus Britton \& Rose (4)

Lophophora J.M.Coult. (2)

Mammillaria Haw. (306)

*Mammillopsis Weber (2)

Mammilloydia Buxb. (2)

Melocactus Link \& Otto (5)

*Mitrocereus (Backeb.) Backeb. (1)

*Morangaya G.D.Rowley (1)

Myrtillocactus Console (3)

Neobesseya Britton \& Rose (2)

*Neobuxbaumia Backeb. (11)

Neolloydia Britton \& Rose (5)

Nopalea Salm-Dyck (7)

Normanbokea Klad. \& Buxb. (1)

Nyctocereus (A.Berger) Britton

\& Rose (3)

*Obregonia Fric ex A.Berger (1)

Oehmea Buxb. (1)

Opuntia Mill. (120)

* Ortegocactus Alexander (1)

*Pachycereus Britton \& Rose (6)

*Pelecyphora Ehrenb. (2)

Peniocereus Britton \& Rose (20)

Pereskia Mill. (4)

Pereskiopsis Britton \& Rose (13)

Pilosocereus Byles \& G.D.Rowley (10)

*Polaskia Backeb. (2)
Pseudorhipsalis Britton \& Rose (1)

* Pterocereus MacDougal \&

Miranda (1)

Rhipsalis Gaertn. (3)

Sclerocactus Britton \& Rose (3)

Selenicereus Britton \& Rose (18)

* Stenocactus (K.Schum.) A.Berger (25)

Stenocereus (A.Berger) Riccob. (21)

* Strombocactus Britton \& Rose (1)

Thelocactus Britton \& Rose (23)

*Turbinicarpus Buxb. \& Backeb. (26)

Werckleocereus Britton \& Rose (1)

Wilcoxia Britton \& Rose (7)

*× Pachgerocereus Moran (1)

Familia Caesalpiniaceae

Bauhinia L. (30)

Caesalpinia L. (37)

Cassia L. (15)

Cercidium Tul. (8)

Cercis L. (1)

Chamaecrista Moench (41)

*Conzattia Rose (2)

Cynometra L. (2)

Dialium L. (1)

Gleditsia L. (1)

Haematoxylum L. (2)

Hoffmannseggia Cav. (11)

Hymenaea L. (1)

Parkinsonia L. (4)

Peltogyne Vogel (1)

Poeppigia C.Presl (1)

Pomaria Cav. (8)

Schizolobium Vogel (1)

Senna Mill. (96)

Swartzia Schreb. (4)

Familia Callitrichaceae

Callitriche L. (6)

Familia Campanulaceae

Burmeistera H.Karst. \& Triana (1)

Calcaratolobelia Wilbur (13)

Campanula L. (1)

Centropogon C.Presl (4)

Diastatea Scheidw. (4)

Downingia Torr. (1)

Githopsis Nutt. (2)

Heterotoma Zucc. (2)

Hippobroma G.Don (1)

Laurentia Adans. (3)

Lobelia L. (62)

Nemacladus Nutt. (10)

Pratia Gaudich. (1)

* Pseudonemacladus McVaugh (1)

Triodanis Raf. ex Greene (3)
Familia Canellaceae

Canella P.Browne (1)

Familia Capparaceae

Atamisquea Miers (1)

Capparis L. (24)

Cleome L. (21)

Cleomella DC. (3)

Crateva L. (3)

Forchhammeria Liebm. (8)

Gynandropsis DC. (1)

Isomeris Nutt. (2)

Koeberlinia Zucc. (1)

Morisonia L. (1)

Polanisia Raf. (5)

Steriphoma Spreng. (1)

Wislizenia Engelm. (3)

Familia Caprifoliaceae

Abelia R.Br. (5)

Lonicera L. (10)

Sambucus L. (2)

Symphoricarpos Ludw. (8)

Viburnum L. (29)

Familia Caricaceae

Carica L. (3)

Jacaratia A.DC. (2)

Jarilla Rusby (3)

Familia Caryophyllaceae

Achyronychia Torr. \& A.Gray (1)

Anychia Michx. (1)

Arenaria L. (27)

Calycotropis Turcz. (1)

Cardionema DC. (1)

Cerastium L. (16)

*Cerdia Moç. \& Sessé ex DC. (2)

Colobanthus Bartl. (2)

Corrigiola Kuntze (1)

Drymaria Willd. (41)

Loeflingia L. (1)

Lychnis L. (1)

Minuartia L. (1)

Paronychia Mill. (9)

Pentacaena Bartl. (1)

Polycarpon L. (1)

Sagina L. (2)

Scopulophila M.E.Jones (1)

Silene L. (9)

Spergularia Pers. (2)

Stellaria L. (8)

Tunica Ludw. (1)

Familia Cecropiaceae

Cecropia L. (4) 
Coussapoa Aubl. (3)

Pourouma Aubl. (2)

\section{Familia Celastraceae}

*Acanthothamnus Brandegee (1)

Canotia Torr. (1)

Celastrus L. (5)

Crossopetalum P.Browne (16)

Elachyptera A.C.Sm. (1)

Elaeodendron Jacq. (3)

Euonymus L. (8)

Gyminda Sarg. (1)

Gymnosporia (Wight \& Arn.)

Hook.f. (1)

Maytenus Molina (8)

Microtropis Wall. (4)

Mortonia A.Gray (6)

Myginda Jacq. (4)

*Orthosphenia Standl. (1)

Paxistima Raf. (1)

Perrottetia Kunth (2)

Quetzalia Lundell (2)

Rhacoma L. (5)

*Rzedowskia Medrano (1)

Schaefferia Jacq. (8)

Tontelea Aubl. (1)

Tricerma Liebm. (2)

Wimmeria Schltdl. \& Cham. (12)

Zinowiewia Turcz. (7)

\section{Familia Ceratophyllaceae}

Ceratophyllum L. (4)

\section{Familia Chenopodiaceae}

Allenrolfea Kuntze (1)

Aphanisma Nutt. ex Moq. (1)

Atriplex L. (39)

Chenopodium L. (18)

Corispermum L. (1)

Cycloloma Moq. (1)

Eurotia Adans. (1)

Meiomeria Standl. (1)

Monolepis Schrad. (2)

Nitrophila S.Watson (1)

Salicornia L. (6)

Sarcobatus Nees (1)

Sarcocornia A.J.Scott (2)

Suaeda Forssk. (16)

Familia Chloranthaceae

Hedyosmum Sw. (1)

Familia Chrysobalanaceae

Chrysobalanus L. (1)

Couepia Aubl. (1)

Hirtella L. (5)
Lecostemon Moç. \& Sessé ex DC. (1)

Licania Aubl. (6)

Familia Cistaceae

Helianthemum Mill. (13)

Lechea L. (3)

\section{Familia Clethraceae}

Clethra L. (17)

\section{Familia Clusiaceae}

Ascyrum L. (1)

Calophyllum L. (1)

Clusia L. (16)

Garcinia L. (2)

Hypericum L. (23)

Mammea L. (1)

Marila Sw. (1)

Rheedia L. (1)

Symphonia L. (1)

Thornea Breedlove \& E.M.McClint. (2)

Tovomita Aubl. (1)

Vismia Vand. (4)

\section{Familia Combretaceae}

Bucida L. (4)

Combretum L. (9)

Conocarpus L. (1)

Laguncularia Gaertn.f. (1)

Terminalia L. (3)

\section{Familia Connaraceae}

Cnestidium Planch. (1)

Connarus L. (4)

Rourea Aubl. (3)

\section{Familia Convolvulaceae}

Aniseia Choisy (2)

Bonamia Thouars (6)

Calycobolus Willd. ex Schult.f. (2)

Calystegia R.Br. (5)

Convolvulus L. (4)

Cressa L. (1)

Dichondra J.R.Forst. \& G.Forst. (7)

Evolvulus L. (20)

Ipomoea L. (162)

Itzaea Standl. \& Steyerm. (1)

Jacquemontia Choisy (20)

Maripa Aubl. (1)

Merremia Dennst. ex Endl. (14)

Odonellia K.R.Robertson (1)

Operculina Manso (3)

Turbina Raf. (1)

\section{Familia Coriariaceae}

Coriaria L. (1)

\section{Familia Cornaceae}

Cornus L. (4)

\section{Familia Crassulaceae}

Crassula L. (5)

Dudleya Britton \& Rose (26)

Echeveria DC. (94)

Graptopetalum Rose (13)

Lenophyllum Rose (4)

* Pachyphytum Link (11)

Sedum L. (101)

* Thompsonella Britton \& Rose (5)

Tillaea L. (2)

Villadia Rose (20)

\section{Familia Crossosomataceae}

Crossosoma Nutt. (3)

Forsellesia Greene (1)

* Velascoa Calderón \& Rzed. (1)

\section{Familia Cucurbitaceae}

*Apatzingania Dieterle (1)

Apodanthera Arn. (8)

Brandegea Cogn. (1)

Cayaponia J.SilvaManso (3)

*Chalema Dieterle (1)

Cionosicyos Benth. \& Hook.f. (2)

Cucurbita L. (19)

Cyclanthera Schrad. (21)

* Dieterlea E.J.Lott (1)

Doyerea Grosourdy (1)

Echinocystis Torr. \& A.Gray (2)

Echinopepon Naudin (13)

Gurania (Schltdl.) Cogn. (1)

Hanburia Seem. (2)

Ibervillea Greene (8)

Luffa Mill. (3)

Marah Kellogg (3)

Melothria P.Browne (3)

Microsechium Naudin (2)

Parasicyos Dieterle (2)

* Peponopsis Naudin (1)

Polyclathra Bertol. (2)

Posadaea Cogn. (1)

Psiguria Neck. ex Arn. (5)

Rytidostylis Hook. \& Arn. (4)

Schizocarpum Schrad. (8)

Sechiopsis Naudin (5)

Sechium P.Browne (6)

Sicydium Schltdl. (5)

Sicyos L. (21)

Sicyosperma A.Gray (1)

Tecunumania Standl. \& Steyerm. (1)

Tumamoca Rose (2)

*Vaseyanthus Cogn. (4) 
Familia Cunoniaceae

Weinmannia L. (4)

Familia Cuscutaceae

Cuscuta L. (58)

Familia Cyrillaceae

Cyrilla L. (1)

Familia Datiscaceae

Datisca L. (1)

Familia Dichapetalaceae

Dichapetalum Thouars (3)

Tapura Aubl. (1)

Familia Dilleniaceae

Curatella Loefl. (1)

Davilla Vand. (3)

Doliocarpus Rol. (1)

Tetracera L. (4)

Familia Droseraceae

Drosera L. (3)

Familia Ebenaceae

Diospyros L. (33)

Familia Elaeocarpaceae

Petenaea Lundell (1)

Sloanea L. (8)

\section{Familia Elatinaceae}

Bergia L. (2)

Elatine L. (3)

Familia Eremolepidaceae

Antidaphne Poepp. \& Endl. (1)

\section{Familia Ericaceae}

Agarista D.Don ex G.Don (4)

Andromeda L. (1)

Arbutus L. (9)

Arctostaphylos Adans. (19)

Befaria Mutis ex L. (5)

Cavendishia Lindl. (6)

Comarostaphylis Zucc. (16)

Disterigma (Klotzsch) Nied. (1)

Empedoclesia Sleumer (2)

Gaultheria L. (17)

Leucothoe D.Don (2)

Lyonia Nutt. (2)

Macleania Hook. (1)

Ornithostaphylos Small (1)

Pernettya Gaud. (4)

Satyria L. (3)
Sphyrospermum Poepp. \& Endl. (1)

Vaccinium L. (14)

Xylococcus Nutt. (1)

\section{Familia Erythroxylaceae}

Erythroxylum L. (12)

Familia Euphorbiaceae

Acalypha L. (114)

Acidocroton Griseb. (2)

Adelia L. (8)

Alchornea Sw. (4)

Andrachne L. (3)

Argythamnia P.Browne (11)

Astrocasia B.L.Rob. \& Millsp. (4)

Bernardia Houst. ex P.Br. (22)

Caperonia A.St.-Hil. (4)

Chiropetalum A.Juss. (2)

Cleidion Blume (1)

Cnidoscolus Pohl (25)

Croton L. (124)

Dalechampia L. (6)

Dalembertia Baill. (4)

Ditaxis Vahl ex Juss. (18)

Drypetes Vahl (3)

* Enriquebeltrania Rzed. (1)

Euphorbia L. (245)

Garcia Rohr (2)

Gymnanthes Sw. (6)

Hippomane L. (1)

Hura L. (1)

Hyeronima Allemao (3)

Jatropha L. (60)

Mabea Aubl. (2)

Manihot Adans. (19)

* Manihotoides J.D.Rogers \& Appan (1)

Margaritaria L. (1)

Meineckia Baill. (1)

Omphalea L. (2)

Pedilanthus Poit. (29)

Pera Mutis (1)

Phyllanthus L. (41)

Piranhea Baill. (1)

Plukenetia L. (3)

Reverchonia A.Gray (1)

Sapium Jacq. (3)

Savia Willd. (1)

Sebastiania Spreng. (16)

Stillingia L. (14)

Tetracoccus Engelm. ex Parry (3)

Tetrorchidium Poepp. \& Endl. (2)

Tragia L. (15)

Familia Fabaceae

Acosmium Schott (1)

Aeschynomene L. (37)
Amicia Kunth (1)

Amorpha L. (4)

Andira Juss. (3)

Apoplanesia C.Presl (1)

Astragalus L. (107)

Ateleia (Moç. \& Sessé ex DC.)

Benth. (8)

Barbieria DC. (1)

Brongniartia Kunth (46)

Calia Berland. (2)

Calopogonium Desv. (4)

Canavalia DC. (15)

Centrosema (DC) Benth. (11)

Chaetocalyx DC. (4)

Clitoria L. (12)

Cologania Kunth (21)

Coursetia DC. (24)

Crotalaria L. (35)

Cymbosema Benth. (1)

Dalbergia L. (16)

Dalea Juss. (192)

Desmodium Desv. (118)

Dioclea Kunth (5)

Diphysa Jacq. (14)

Dussia Krug \& Urb. ex Taub. (2)

Eriosema (DC.) Rchb. (14)

Errazurizia Phil. (2)

Erythrina L. (28)

Eysenhardtia Kunth (11)

Galactia P.Browne (16)

Genistidium I.M.Johnst. (1)

Gliricidia Kunth (3)

Glycyrrhiza L. (1)

Harpalyce Sessé \& Moç. ex DC. (8)

* Hesperothamnus Brandegee (5)

Hoita Rydb. (2)

Hybosema Harms (2)

Indigofera L. (31)

Lathyrus L. (9)

Lennea Klotzsch (3)

Lespedeza Michx. (3)

Lonchocarpus Kunth (67)

Lotus L. (35)

Lupinus L. (82)

Machaerium Pers. (18)

Macroptilium (Benth.) Urb. (6)

Marina Liebm. (40)

Minkelersia M. Martens \& Galeotti (2)

Mucuna Adans. (5)

Muellera L. (2)

Myrospermum Jacq. (1)

Myroxylon L.f. (4)

Nissolia Jacq. (14)

Olneya A.Gray (1)

Orbexilum Raf. (1)

Ormosia Jack. (6) 
Orobus L. (1)

Oxyrhynchus Brandegee (3)

Pachecoa Standl. \& Steyerm. (1)

Pachyrhizus Rich. ex DC. (4)

Pediomelum Rydb. (5)

Petalostemon Michx. (6)

Peteria A.Gray (3)

Phaseolus L. (56)

Pickeringia Nutt. ex Torr. \& A.Gray (1)

Piscidia L. (6)

Platymiscium Vogel (6)

Poiretia Vent. (1)

Psoralidium Rydb. (1)

Psorothamnus Rydb. (5)

Pterocarpus L. (4)

Ramirezella Rose (10)

Rhynchosia Lour. (27)

Robinia L. (4)

Rupertia Grimes (1)

Sesbania Scop. (4)

Sophora L. (3)

Sphinctospermum Rose (1)

Stizolobium P.Browne (2)

Strophostyles Elliott (1)

Stylosanthes Sw. (8)

Styphnolobium Schott ex Endl. (3)

Tephrosia Pers. (50)

Teramnus P.Browne (2)

Trifolium L. (21)

Uribea Dugand \& Romero (1)

Vatairea Aubl. (1)

Vicia L. (10)

Vigna Savi (18)

Willardia Rose (3)

Zornia J.F.Gmel. (9)

\section{Familia Fagaceae}

Castanea Mill. (1)

Fagus L. (1)

Quercus L. (192)

\section{Familia Flacourtiaceae}

Abatia Ruiz \& Pav. (1)

Banara Aubl. (1)

Bartholomaea Standl. \& Steyerm. (1)

Casearia Jacq. (22)

*Chiangiodendron T.Wendt (1)

Dendrostylis H.Karst. \& Triana (1)

Hasseltia Kunth (2)

Hasseltiopsis Sleumer (1)

Homalium Jacq. (4)

Laetia Loefl. ex L. (1)

Lunania Hook. (1)

Muntingia L. (1)

Neopringlea S.Watson (3)

Olmediella Baill. (1)
Oncoba Forssk. (1)

Pleuranthodendron L.O.Williams (1)

Prockia S.Watson (1)

Samyda L. (2)

Xylosma G.Forst. (13)

Zuelania A.Rich. (1)

Familia Fouquieriaceae

Fouquieria Kunth (13)

Familia Frankeniaceae

Frankenia L. (6)

Familia Fumariaceae

Corydalis DC. (4)

\section{Familia Garryaceae}

Garrya Douglas (12)

\section{Familia Gentianaceae}

Centaurium Hill (21)

Chelonanthus (Griseb.) Gilg (1)

Coutoubea Aubl. (1)

Curtia Cham. \& Schltdl. (1)

Erythraea Borkh. (1)

Eustoma Salisb. (4)

* Geniostemon Engelm. \& A.Gray (3)

Gentiana L. (19)

Gentianella Moench (6)

Gentianopsis Ma (5)

Halenia Borkh. (14)

Leiphaimos Schltdl. \& Cham. (2)

Lisianthus P.Browne (9)

Sabatia Adans. (3)

Schultesia Mart. (3)

Swertia L. (2)

Voyria Aubl. (5)

Familia Geraniaceae

Erodium L'Her. (4)

Geranium L. (40)

\section{Familia Gesneriaceae}

Achimenes Pers. (27)

Alloplectus Mart. (5)

Besleria L. (7)

Capanea Decne. ex Planch. (1)

Codonanthe (Mart.) Hanst. (3)

Columnea L. (10)

Diastema Benth. (2)

Drymonia Mart. (4)

Episcia Mart. (6)

Eucodonia Hanst. (2)

Koellikeria Regel (1)

Kohleria Regel (7)

Monopyle Moritz ex Benth. (1)
Moussonia Regel (7)

Napeanthus Gardner (2)

Nautilocalyx Linden ex Hanst. (1)

Neomortonia Wiehler (1)

Niphaea Lindl. (1)

Oerstedina Wiehler (1)

Phinaea Benth. (3)

Rechsteineria Regel (1)

Rhynchoglossum Blume (1)

Sinningia Nees (2)

* Smithiantha Kuntze (5)

Solenophora Benth. (12)

Familia Goodeniaceae

Scaevola L. (1)

Familia Grossulariaceae

Fendlera Engelm. \& A.Gray (3)

Fendlerella Heller (3)

Grossularia Mill. (4)

Phyllonoma Willd. (1)

* Pterostemon Schauer (3)

Ribes L. (20)

Familia Gunneraceae

Gunnera L. (3)

Familia Haloragaceae

Myriophyllum L. (6)

Proserpinaca L. (2)

Familia Hamamelidaceae

Liquidambar L. (1)

Matudaea Lundell (1)

Molinadendron P.K.Endress (1)

Familia Hernandiaceae

Gyrocarpus Jacq. (2)

Hernandia L. (4)

Sparattanthelium Mart. (1)

Familia Hippocastanaceae

Aesculus L. (1)

Billia Peyr. (1)

Familia Hippocrateaceae

Hippocratea L. (13)

Salacia L. (4)

Familia Hippuridaceae

Hippuris L. (1)

Familia Hydrangeaceae

Deutzia Thunb. (3)

Hydrangea L. (4)

Jamesia Torr. \& A.Gray (1)

Philadelphus L. (17) 
Familia Hydrophyllaceae

Emmenanthe Benth. (1)

Eriodictyon Benth. (4)

Eucrypta Nutt. (3)

Hydrolea L. (4)

Nama L. (44)

Nemophila Buckley (4)

Phacelia Juss. (59)

Pholistoma Lilja (3)

Turricula J.F.Macbr. (1)

Wigandia Kunth (1)

\section{Familia Icacinaceae}

Calatola Standl. (4)

Mappia Jacq. (3)

Oecopetalum Greenm. \&

C.H.Thomps. (3)

Ottoschulzia Urb. (1)

Familia Illiciaceae

Illicium L. (3)

\section{Familia Juglandaceae}

Alfaroa Standl. (2)

Carya Nutt. (5)

Engelhardtia Blume (1)

Juglans L. (7)

Familia Julianiaceae

Amphipterygium Schiede (5)

Familia Krameriaceae

Krameria Loefl. (12)

Familia Lacistemataceae

Lacistema Sw. (1)

Lozania Mutis (1)

\section{Familia Lamiaceae}

Acanthomintha (A.Gray) A.Gray (1)

Agastache J.Clayton ex Gronov. (18)

*Asterohyptis Epling (3)

Catopheria (Benth.) Benth. (2)

Chaunostoma Donn.Sm. (1)

Cunila L. (8)

*Eplingia L.O.Williams (1)

Gardoquia Ruiz \& Pav. (3)

Hedeoma Juss. (32)

Hesperozygis Epling (1)

Hyptis Jacq. (42)

Lepechinia Willd. (6)

Marsypianthes Mart. ex Benth. (1)

Monarda L. (8)

Monardella Benth. (7)

Neoeplingia Ramamoorthy, Hiriart \& Medrano (1)
Physostegia Benth. (3)

Pogogyne Benth. (3)

Poliomintha A.Gray (9)

Prunella L. (2)

Salazaria Torr. (1)

Salvia L. (292)

Satureja L. (16)

Scutellaria L. (45)

Solenostemon Thonn. (1)

Sphacele Benth. (2)

Stachys L. (35)

Tetraclea A.Gray (3)

Teucrium L. (10)

Trichostema L. (7)

Warnockia M.W.Turner (1)

\section{Familia Lauraceae}

Aiouea Aubl. (1)

Beilschmiedia Nees (8)

Cassytha L. (1)

Cinnamomum Schaeff. (21)

Cryptocarya R.Br. (1)

Licaria Aubl. (16)

Litsea Lam. (7)

*Mocinnodaphne Lorea-Hern. (1)

Nectandra Roland (30)

Ocotea Aubl. (26)

Persea Gaertn.f. (19)

Phoebe Nees (20)

Umbellularia Nutt. (1)

Familia Lecythidaceae

Eschweilera Mart. ex DC. (1)

Familia Lennoaceae

Lennoa La Llave (1)

Pholisma Nutt. (3)

Familia Lentibulariaceae

Genlisea A.St.-Hil. (1)

Pinguicula L. (38)

Utricularia L. (18)

\section{Familia Linaceae}

Linum L. (26)

\section{Familia Loasaceae}

Cevallia Lag. (1)

Eucnide Zucc. (13)

Gronovia L. (2)

Klaprothia Kunth (1)

Loasa Adans. (1)

Mentzelia L. (34)

Petalonyx A.Gray (3)

*Schismocarpus S.F.Blake (2)
Familia Loganiaceae

Cynoctonum J.F.Gmel. (2)

Gelsemium Juss. (1)

Polypremum L. (1)

Spigelia L. (12)

Strychnos L. (7)

Cladocolea Tiegh. (15)

Familia Loranthaceae

Oryctanthus Eichl. (3)

Phthirusa Mart. (1)

Psittacanthus Mart. (10)

Struthanthus Mart. (24)

Familia Lythraceae

Adenaria Kunth (1)

Ammannia Scop. (3)

Cuphea P.Browne (104)

Ginoria Jacq. (1)

Heimia Link (1)

Lafoensia Vand. (1)

Lythrum L. (7)

Nesaea Comm. ex Kunth (1)

Rotala L. (2)

Familia Magnoliaceae

Magnolia L. (10)

Talauma Juss. (1)

Familia Malpighiaceae

Aspicarpa Rich. (5)

Banisteria L. (3)

Banisteriopsis C.B.Rob. (4)

Brachypteris Griseb. (1)

Bunchosia Rich. ex Juss. (19)

Byrsonima Rich. ex Kunth (2)

Callaeum Small (6)

Diplopterys A.Juss. (1)

*Echinopterys Juss. (2)

Galphimia Cav. (11)

Gaudichaudia Kunth (15)

Heteropterys Kunth (8)

Hiraea Jacq. (6)

Janusia Juss. (3)

Lasiocarpus Liebm. (3)

Malpighia L. (23)

Mascagnia Bertero (15)

Stigmaphyllon Juss. (7)

Tetrapterys Cav. (11)

Familia Malvaceae

Abutilon Mill. (39)

Allosidastrum (Hochr.) Krapov.,

Fryxell \& D.M.Bates (3)

Allowissadula Bates (9)

Anoda Cav. (24) 
*Anotea (DC.) Kunth (1)

Bakeridesia Hochr. (10)

Bastardia Kunth (2)

* Bastardiastrum (Rose) Bates (8)

Batesimalva Fryxell (3)

Billieturnera Fryxell (1)

Briquetia Hochr. (3)

Callirhoe Nutt. (2)

Cienfuegosia Cav. (3)

* Dendrosida Fryxell (5)

Dirhamphis Krapov. (1)

Eremalche Greene (2)

Fryxellia Bates (1)

Gaya Kunth (3)

Gossypium L. (12)

Hampea Schltdl. (11)

Herissantia Medik. (2)

Hibiscus L. (32)

Hochreutinera Krapov. (1)

Horsfordia A.Gray (4)

Kearnemalvastrum Bates (2)

Kosteletzkya C.Presl (8)

Krapovickasia Fryxell (1)

Lavatera L. (3)

Lopimia Martius (1)

Malachra L. (4)

Malacothamnus Greene (4)

Malvastrum A.Gray (8)

Malvaviscus Fabr. (7)

Malvella Jaub. \& Spach (3)

Meximalva Fryxell (2)

Neobrittonia Hochr. (1)

Pavonia Cav. (32)

Peltaea (C.Presl) Standl. (1)

Periptera DC. (5)

Phymosia Ham. (7)

Pseudabutilon R.E.Fr. (5)

Rhynchosida Fryxell (1)

Robinsonella Rose \& E.G.Baker (15)

Sida L. (35)

Sidalcea A.Gray (2)

Sidastrum Baker f. (5)

Sphaeralcea A.St.-Hil. (21)

Tarasa Phil. (2)

Thespesia Sol. ex Correa (1)

Urena L. (1)

Urocarpidium Ulbr. (2)

Wissadula Medik. (3)

Familia Marcgraviaceae

Marcgravia L. (5)

Ruyschia Jacq. (1)

Souroubea Aubl. (3)

Familia Melastomataceae

Aciotis D.Don (1)
Acisanthera P.Browne (2)

Adelobotrys A.DC. (1)

Arthrostemma Ruiz \& Pav. (4)

Bellucia Neck. (4)

Blakea P.Browne (1)

Centradenia G.Don (3)

Clidemia D.Don (24)

Conostegia D.Don (12)

Graffenrieda C.Mart. (1)

Henriettea A.DC. (3)

Heterocentron Hook. \& Arn. (10)

Heterotrichum DC. (1)

Leandra Raddi (6)

Maieta Aubl. (1)

Meriania Sw. (1)

Miconia Ruiz \& Pav. (92)

Monochaetum Naud. (7)

Mouriri Aubl. (4)

Ossaea A.DC. (2)

Pterolepis Miq. (3)

Rhynchanthera DC. (3)

Schwackaea Cogn. (1)

Stanmarkia Almeda (1)

Tibouchina Aubl. (18)

Tococa Aubl. (1)

Topobea Aubl. (4)

Triolena Naud. (1)

Familia Meliaceae

Cedrela L. (7)

Guarea F.Allam. ex L. (4)

Swietenia Jacq. (2)

Trichilia L. (19)

Familia Menispermaceae

Abuta Aubl. (2)

Cissampelos L. (4)

Cocculus DC. (2)

Disciphania Eichler (4)

Hyperbaena Miers (4)

Menispermum L. (1)

Odontocarya Miers (1)

Familia Menyanthaceae

Nymphoides Hill (3)

Familia Mimosaceae

Abarema Pittier (2)

Acacia Mill. (92)

Acaciella Britton \& Rose (7)

Adenopodia C.Presl (4)

Albizia Durazz. (5)

Balizia Barneby \& J.W.Grimes (1)

Calliandra Benth. (48)

*Calliandropsis H.M.Hern. \& P.Guinet (1)
Chloroleucon Britton \& Rose (1)

Cojoba Britton \& Rose (5)

Desmanthus Willd. (18)

Ebenopsis Britton \& Rose (3)

Entada Adans. (3)

Entadopsis Britton (1)

Enterolobium Mart. (2)

* Guinetia L.Rico \& M.Sousa (1)

Havardia Small (5)

Hesperalbizia Barneby \&

J.W.Grimes (1)

Inga Mill. (49)

Leucaena Benth. (25)

Lysiloma Benth. (11)

Microlobius C.Presl (1)

Mimosa L. (138)

Neptunia Lour. (4)

Painteria Britton \& Rose (3)

Piptadenia Benth. (3)

Pithecellobium Mart. (10)

Pityrocarpa (Benth.) Britton \& Rose (1)

Prosopidastrum L. (1)

Prosopis Burkart (9)

Schrankia Hassl. (9)

Sphinga Barneby \& J.W.Grimes (2)

Zapoteca H.M.Hern. (17)

Zygia P.Browne (7)

Familia Mitrastemonaceae

Mitrastemon Makino (1)

Familia Molluginaceae

Glinus L. (1)

Familia Monimiaceae

Mollinedia Ruiz \& Pav. (11)

Siparuna Aubl. (5)

Familia Monotropaceae

Monotropa L. (3)

Pterospora Nutt. (1)

\section{Familia Moraceae}

Brosimum Sw. (5)

Castilla Cerv. (1)

Clarisia Ruiz \& Pav. (1)

Dorstenia L. (9)

Ficus L. (34)

Maclura Nutt. (3)

Morus L. (2)

Poulsenia Eggers (1)

Pseudolmedia Trécul (3)

Sorocea A.St.-Hil. (1)

Trophis L. (5)

Familia Myricaceae

Myrica L. (4) 
Familia Myristicaceae

Compsoneura Warb. (1)

Virola Aubl. (3)

\section{Familia Myrsinaceae}

Amatlania Lundell (5)

Ardisia Sw. (30)

Gentlea Lundell (7)

Heberdenia Banks ex A.DC. (1)

Ibarraea Lundell (6)

Myrsine L. (6)

Oerstedianthus Lundell (6)

Parathesis Hook.f. (56)

Stylogyne DC. (3)

Synardisia (Mez) Lundell (1)

Yunckeria Lundell (2)

Zunila Lundell (6)

\section{Familia Myrtaceae}

Acca O.Berg (1)

Calycorectes O.Berg (1)

Calyptranthes Sw. (21)

Chamguava Landrum (2)

Eugenia L. (80)

Mosiera Small (2)

Myrcia DC. (2)

Myrcianthes O.Berg (1)

Myrciaria O.Berg (1)

Pimenta Lindl. (2)

Psidium L. (9)

Ugni Turcz. (2)

Familia Nelumbonaceae

Nelumbo Adans. (1)

Familia Nyctaginaceae

Abronia Juss. ex Lam. (10)

Acleisanthes A.Gray (6)

Allionia L. (7)

Ammocodon Standl. (1)

Anulocaulis Standl. (2)

Boerhavia L. (22)

Boldoa Cav. ex Lag. (1)

Commicarpus Standl. (2)

Cyphomeris Standl. (2)

Grajalesia Miranda (1)

Guapira Aubl. (3)

Mirabilis L. (36)

Neea Ruiz \& Pav. (10)

Nyctaginia Choisy (1)

Okenia Schltdl. \& Cham. (1)

Oxybaphus L'Her. ex Willd. (4)

Pisonia L. (4)

Pisoniella (Heimerl) Standl. (1)

Salpianthus Humb. \& Bonpl. (3)

Selinocarpus A.Gray (7)
Torrubia Vell. (1)

Tripterocalyx Hook. ex Standl. (1)

Familia Nymphaeaceae

Nuphar Sm. (1)

Nymphaea L. (12)

Familia Nyssaceae

Nyssa L. (1)

Familia Ochnaceae

Ouratea Aubl. (12)

Sauvagesia L. (3)

Familia Olacaceae

Heisteria Jacq. (3)

Schoepfia Schreb. (4)

Ximenia L. (4)

\section{Familia Oleaceae}

Chionanthus L. (2)

Forestiera Poir. (11)

Fraxinus L. (22)

*Hesperelaea A.Gray (1)

Linociera Sw. ex Schreb. (2)

Menodora Humb. \& Bonpl. (16)

Osmanthus Lour. (2)

Familia Onagraceae

Calylophus Spach (9)

Camissonia Link (27)

Clarkia Pursh (5)

Diplandra Hook. \& Arn. (1)

Epilobium L. (11)

Fuchsia L. (28)

Gaura L. (13)

Gayophytum A.Juss. (1)

Gongylocarpus Schltdl. \& Cham. (4)

Hauya DC. (7)

Jussiaea L. (3)

Lopezia Cav. (27)

Ludwigia L. (20)

*Megacorax S.González \&

W.L.Wagner (1)

Oenothera L. (54)

Semeiandra Hook. \& Arn. (1)

*Xylonagra Donn.Sm. \& Rose (2)

\section{Familia Opiliaceae}

Agonandra Miers (4)

Familia Orobanchaceae

Castilleja Mutis (80)

Conopholis Wallr. (1)

Orobanche L. (9)
Familia Oxalidaceae

Biophytum A.DC. (2)

Oxalis L. (41)

Familia Papaveraceae

Argemone L. (22)

Bocconia L. (8)

Dendromecon Benth. (1)

Eschscholzia Cham. (8)

*Hunnemannia Sweet (2)

Meconella Nutt. ex Torr. \& A.Gray (1)

Platystemon Benth. (2)

Romneya Harv. (2)

Stylomecon G.Taylor (1)

Familia Passifloraceae

Passiflora L. (91)

Familia Pedaliaceae

Martynia L. (1)

Proboscidea Adans. (8)

Familia Phytolaccaceae

Agdestis Moç. \& Sessé ex DC. (1)

Ledenbergia Klotzsch ex Moq. (1)

*Nowickea J.Martínez \& J.A.

McDonald (2)

Petiveria L. (1)

Phytolacca L. (5)

Rivina L. (1)

Stegnosperma Benth. (3)

Trichostigma A.Rich. (1)

Familia Piperaceae

Peperomia Ruiz \& Pav. (116)

Piper L. (153)

Pothomorphe Miq. (1)

Familia Plantaginaceae

Plantago L. (14)

Familia Platanaceae

Platanus L. (7)

Familia Plumbaginaceae

Limonium Mill. (2)

Plumbago L. (2)

Familia Podostemaceae Marathrum Humb. \& Bonpl. (3)

Oserya Tul. \& Wedd. (2)

Podostemum Michx. (1)

Tristicha Thouars (1)

* Vanroyenella Novelo \& C.T.Philbrick (1) 
Familia Polemoniaceae

*Acanthogilia A.G.Day \& Moran (1)

Allophyllum (Nutt.) V.E.Grant

\& A.D.Grant (2)

Bonplandia Cav. (1)

Cobaea Cav. (9)

Eriastrum Wooton \& Standl. (8)

Gilia Ruiz \& Pav. 27

Ipomopsis Michx. (18)

Langloisia Greene (3)

Leptodactylon Hook. \& Arn. (2)

Linanthus Benth. (16)

Loeselia L. (9)

Navarretia Ruiz \& Pav. (6)

Phlox L. (7)

Polemonium L. (4)

Sympetaleia A.Gray (3)

Familia Polygalaceae

Bredemeyera Willd. (1)

Monnina Ruiz \& Pav. (7)

Polygala L. (101)

Securidaca L. (3)

Familia Polygonaceae

Antigonon Endl. (4)

Centrostegia A.Gray ex Benth. (1)

Chorizanthe R.Br. ex Benth. (17)

Coccoloba L. (32)

Eriogonum Michx. (63)

Gymnopodium Rolfe (1)

* Harfordia Greene \& Parry (3)

Lastarriaea Remy (3)

Muehlenbeckia Meisn. (2)

Nemacaulis Nutt. (1)

Neomillspaughia S.F.Blake (1)

Oxytheca Nutt. (1)

Podopterus Humb. \& Bonpl. (2)

Polygonum L. (28)

Pterostegia Fisch. \& Mey. (1)

Rumex L. (12)

Ruprechtia C.A.Mey. (7)

Triplaris L. (4)

\section{Familia Portulacaceae}

Calandrinia Kunth (2)

Cistanthe Spach (6)

Claytonia L. (2)

Lewisia Pursh (2)

Montia L. (3)

Phemeranthus Raf. (4)

Portulaca L. (11)

Talinaria Brandegee (1)

Talinopsis A.Gray (1)

Talinum Adans. (17)
Familia Primulaceae

Anagallis L. (1)

Androsace L. (2)

Dodecatheon L. (3)

Lysimachia L. (2)

Primula L. (3)

Samolus L. (9)

Familia Proteaceae

Roupala Aubl. (2)

Familia Pyrolaceae

Chimaphila Pursh (3)

Pyrola L. (5)

Sarcodes Torr. (1)

Familia Rafflesiaceae

Apodanthes Poit. (1)

*Bdallophyton Eichler (4)

Pilostyles Guill. (3)

Familia Ranunculaceae

Aconitum L. (1)

Anemone L. (5)

Aquilegia L. (7)

Clematis L. (16)

Delphinium L. (22)

Myosurus L. (2)

Paeonia L. (1)

Ranunculus L. (31)

Thalictrum L. (26)

\section{Familia Resedaceae}

Oligomeris Cambess. (1)

Familia Rhamnaceae

Adolphia Meisn. (2)

Ceanothus L. (22)

Colubrina Rich. ex Brongn. (19)

Condalia Cav. (17)

Condaliopsis (Weberb.) Suess. (2)

Gouania Jacq. (6)

Karwinskia Zucc. (10)

Krugiodendron Urb. (1)

Rhamnus L. (32)

Sageretia Brongn. (4)

Ziziphus Adans. (8)

Familia Rhizophoraceae

Cassipourea Aubl. (1)

Rhizophora L. (2)

Familia Rosaceae

Acaena Mutis ex L. (1)

Adenostoma Hook. \& Arn. (3)

Agrimonia L. (1)
Alchemilla L. (16)

Amelanchier Medik. (5)

Aphanes L. (1)

Cercocarpus Kunth (13)

Chamaebatia Benth. (1)

Crataegus L. (14)

Fallugia Endl. (1)

Fragaria L. (3)

Geum L. (3)

Guamatela Donn.Sm. (1)

Heteromeles M.Roem. (2)

Holodiscus (K.Koch) Maxim. (6)

Horkelia Cham. \& Schltdl. (3)

Ivesia Torr. \& A.Gray (1)

* Lindleya Kunth (1)

Petrophytum Rydb. (1)

Photinia Lindl. (6)

Physocarpus Maxim. (1)

Potentilla Adans. (30)

Prunus L. (29)

Purshia DC. (5)

Rosa L. (13)

Rubus L. (41)

Sericotheca Raf. (6)

Sibbaldia L. (1)

Vauquelinia Correa ex Humb.

\& Bonpl. (8)

*Xerospiraea Henr. (1)

Familia Rubiaceae

Alibertia A.Rich. ex DC. (1)

Allenanthus Standl. (1)

Alseis Schott (2)

Amaioua Aubl. (1)

Anisomeris C.Presl (4)

Antirhea A.DC. (2)

Appunia Hook.f. (1)

Asemnantha Hook.f. (1)

Augusta Pohl (2)

Balmea Martínez (1)

Bertiera Aubl. (1)

Blepharidium Standl. (1)

Bouvardia Salisb. (33)

Calycophyllum DC. (1)

Carterella Terrell (1)

Cephaelis Sw. (3)

Cephalanthus L. (2)

Chiococca P.Browne (12)

Chione DC. (3)

Chomelia Jacq. (3)

*Cigarrilla Aiello (1)

Coccocypselum P.Browne (5)

Cosmibuena Ruiz \& Pav. (2)

*Cosmocalyx Standl. (1)

Coussarea Aubl. (2)

Coutaportla Urb. (3) 
Coutarea Aubl. (1)

Crusea Cham. \& Schltdl. (16)

Declieuxia Kunth (1)

Deppea Schltdl. \& Cham. (23)

Didymaea Hook.f. (4)

Diodia L. (8)

Eizia Standl. (1)

Elaeagia Wedd. (1)

Erithalis P.Browne (1)

Ernodea Sw. (1)

Exostema (Pers.) Rich. ex Humb.

\& Bonpl. (3)

Faramea Aubl. (5)

Galianthe Griseb. (1)

Galium L. (48)

Genipa L. (2)

Geophila D.Don (2)

Glossostipula Lorence (2)

Gonzalagunia Ruiz \& Pav. (5)

Guettarda L. (8)

*Habroneuron Standl. (1)

Hamelia Jacq. (10)

Hedyotis L. (16)

Hemidiodia K.Schum. (1)

Hillia Jacq. (5)

Hintonia Bullock (4)

Hoffmannia Sw. (36)

Houstonia L. (12)

Isertia Schreb. (1)

Ixora L. (1)

Lindenia Benth. (1)

Machaonia Humb. \& Bonpl. (7)

Manettia L. (2)

Mitchella L. (1)

Mitracarpus Zucc. (3)

Morinda L. (4)

Nertera Banks \& Sol. ex Gaertn. (1)

Oldenlandia L. (2)

*Omiltemia Standl. (2)

*Otocalyx Brandegee (1)

Paederia L. (2)

Palicourea Aubl. (9)

Pinarophyllon Brandegee (2)

Pittoniotis Griseb. (1)

* Placocarpa Hook.f. (1)

Pogonopus Klotzsch (1)

Portlandia P.Browne (2)

Posoqueria Aubl. (2)

Psychotria L. (85)

Rachicallis DC. (1)

Randia L. (48)

Ravnia Oerst. (1)

Relbunium (Endl.) Hook.f. (1)

Richardia L. (4)

Rondeletia L. (63)

Rudgea Salisb. (1)
Sabicea Aubl. (3)

Sickingia Willd. (1)

Simira Aubl. (4)

Sommera Schltdl. (7)

Spermacoce L. (21)

Staelia Cham. \& Schltdl. (1)

* Stenotis Terrell (8)

Steyermarkia Standl. (1)

Strumpfia Jacq. (1)

Stylosiphonia Brandegee (1)

*Syringantha Standl. (1)

\section{Familia Rutaceae}

Amyris L. (15)

Casimiroa La Llave (8)

Choisya Kunth (5)

Cneoridium Hook.f. (1)

Decatropis Hook.f. (2)

Decazyx Pittier \& S.F.Blake (1)

Ertela Adans. (1)

Erythrochiton Nees \& Mart. (1)

Esenbeckia Kunth (11)

Galipea Aubl. (2)

Helietta Tul. (3)

Megastigma Hook.f. (2)

Peltostigma Walp. (1)

Pilocarpus Vah(2)

* Polyaster Hook.f. (1)

Ptelea L. (2)

*Stauranthus Liebm. (2)

Thamnosma Torr. \& Frem. (5)

Zanthoxylum L. (41)

Familia Sabiaceae

Meliosma Blume (11)

Familia Salicaceae

Populus L. (18)

Salix L. (25)

Familia Santalaceae

Comandra Nutt. (1)

Familia Sapindaceae

Allophylus L. (4)

Averrhoidium Baill. (1)

Blomia Miranda (2)

Cardiospermum L. (6)

Cupania L. (14)

Dodonaea Jacq. (1)

Exothea Macfad. (3)

Matayba Aubl. (7)

Melicoccus P.Browne (1)

Paullinia L. (15)

Sapindus L. (1)

Serjania Schumach. (55)
Talisia Aubl. (4)

Thinouia Triana \& Planch. (2)

Thouinia Poit. (4)

Thouinidium Radlk. (2)

Ungnadia Endl. (1)

Urvillea Kunth (2)

Familia Sapotaceae

Chrysophyllum L. (2)

Manilkara Adans. (2)

Micropholis (Griseb.) Pierre (1)

Peteniodendron Lundell (1)

Pouteria Aubl. (13)

Sideroxylon L. (27)

Familia Saururaceae

Anemopsis Hook. \& Arn. (1)

Familia Saxifragaceae

Heuchera L. (10)

Jepsonia Small (1)

Lithophragma (Nutt.) Torr. \& A.Gray (4)

Saxifraga L. (2)

Familia Schisandraceae

Schisandra Michx. (1)

Familia Scrophulariaceae

Agalinis Raf. (10)

Alectra Thunb. (1)

Alonsoa Ruiz \& Pav. (1)

*Amalophyllon Brandegee (1)

Angelonia Humb. \& Bonpl. (2)

Antirrhinum L. (8)

Asarina Mill. (3)

Aureolaria Raf. (1)

Bacopa Aubl. (8)

Benjaminia Vell. (1)

Brachystigma Pennell (1)

Buchnera L. (8)

Calceolaria L. (5)

Capraria L. (4)

* Clevelandia Greene (1)

Collinsia Nutt. (3)

Cordylanthus Nutt. ex Benth. (6)

Eremogeton Standl. \& L.O.Williams (1)

Escobedia Ruiz \& Pav. (6)

Galvezia Dombey ex A.Juss. (4)

* Gentrya Breedlove \& Heckard (1)

Gibsoniothamnus L.O.Williams (2)

Hemichaena Benth. (5)

Ilysanthes Raf. (1)

Keckiella Straw (4)

Lamourouxia Kunth (24)

Leucocarpus D.Don (1)

Leucophyllum Humb. \& Bonpl. (14) 
Limosella L. (1)

Linaria Mill. (4)

Lindernia All. (7)

Lophospermum D.Don (8)

Mabrya Elisens (5)

Maurandya Ortega (8)

Mecardonia Ruiz \& Pav. (3)

Melasma Berg (1)

Micranthemum Michx. (2)

Mimulus L. (31)

Mohavea A.Gray (1)

*Ophiocephalus Wiggins (1)

Pedicularis L. (10)

Penstemon Schmidel (65)

Pseudorontium (A.Gray) Rothm. (1)

*Rhodochiton Zucc. ex Otto

\& D.Dietr. (1)

Russelia Jacq. (43)

Schistophragma Benth. (5)

Scoparia L. (3)

Scrophularia L. (2)

Seymeria Pursh (16)

Sibthorpia L. (2)

* Silviella Pennell (2)

Stemodia L. (17)

Tetranema Benth. ex Lindl. (2)

Uroskinnera Lindl. (4)

Veronica L. (6)

Familia Setchellanthaceae

* Setchellanthus Brandegee (1)

Familia Simaroubaceae

Alvaradoa Liebm. (1)

Castela Turpin (6)

Picramnia Sw. (11)

Picrasma Blume (1)

Picrella Baill. (1)

Quassia L. (1)

*Recchia Moç. \& Sessé ex DC. (3)

Simarouba Aubl. (1)

\section{Familia Simmondsiaceae}

Simmondsia Nutt. (1)

Familia Solanaceae

Acnistus Schott (3)

Athenaea Sendtn. (4)

Bouchetia Dunal (1)

Brachistus Miers (2)

Browallia L. (2)

Brugmansia Pers. (2)

Brunfelsia L. (1)

Capsicum L. (10)

Cestrum L. (36)

Chamaesaracha (A.Gray) Benth. (7)
Cyphomandra C.Mart. ex G.Sendtn. (2)

Datura L. (11)

Grabowskia Schltdl. (1)

Hunzikeria D'Arcy (1)

Jaltomata Schltdl. (3)

Juanulloa Ruiz \& Pav. (1)

Lycianthes (Dunal) Hassl. (34)

Lycium L. (31)

Markea Rich. (2)

Melananthus Walp. (1)

Nectouxia Kunth (1)

Nicotiana L. (10)

Nierembergia Ruiz \& Pav. (1)

Petunia A.Juss. (4)

Physalis L. (91)

Quincula Raf. (1)

Salpiglossis Ruiz \& Pav. (1)

Schultesianthus Hunz. (1)

Schwenckia Vahl (1)

Solandra Sw. (8)

Solanum L. (168)

Tzeltalia E.Estrada \& M.Martínez (2)

Witheringia Miers (11)

\section{Familia Staphyleaceae}

Huertea Ruiz \& Pav. (1)

Staphylea L. (1)

Turpinia Vent. (6)

\section{Familia Sterculiaceae}

Ayenia L. (24)

Byttneria L. (3)

Chiranthodendron Larreat. (1)

Fremontodendron Coville (2)

Guazuma Adans. (1)

Helicteres L. (4)

Hermannia L. (4)

Melochia L. (18)

* Physodium C.Presl (5)

Sterculia L. (3)

Theobroma L. (2)

Waltheria L. (17)

\section{Familia Styracaceae}

Styrax L. (14)

Familia Surianaceae

Suriana L. (1)

Familia Symplocaceae

Symplocos Jacq. (21)

Familia Theaceae

Cleyera Thunb. (5)

Freziera Willd. (3)

Laplacea Kunth (1)
Symplococarpon Airy Shaw (2)

Ternstroemia Mutis ex L.f. (11)

Familia Theophrastaceae

Deherainia Decne. (3)

Jacquinia L. (10)

Familia Thymelaeaceae

Daphnopsis Mart. \& Zucc. (17)

Dirca L. (1)

Familia Ticodendraceae

Ticodendron Gómez-Laurito \&

L.D.Gómez (1)

Familia Tiliaceae

Apeiba Aubl. (1)

Carpodiptera Griseb. (1)

Christiana A.DC. (1)

Corchorus L. (3)

Heliocarpus L. (13)

Luehea Willd. (3)

Mortoniodendron Standl. \&

Steyerm. (5)

Tilia L. (1)

Trichospermum Blume (3)

Triumfetta L. (36)

Familia Tovariaceae

Tovaria Ruiz \& Pav. (1)

Familia Trigoniaceae

Trigonia Aubl. (2)

Familia Tropaeolaceae

Tropaeolum L. (1)

Familia Turneraceae

Erblichia Seem. (1)

Piriqueta Aubl. (4)

Turnera L. (6)

\section{Familia Ulmaceae}

Ampelocera Klotzsch (1)

Celtis L. (8)

Lozanella Greenm. (1)

Phyllostylon Capan. ex Benth. \&

Hook. (1)

Trema Lour. (4)

Ulmus L. (6)

\section{Familia Urticaceae}

Boehmeria Jacq. (9)

Discocnide Chew (1)

Hemistylis Walp. (1)

Hesperocnide Torr. (1) 
Laportea Gaudich. (1)

Myriocarpa Benth. (6)

Parietaria L. (4)

Phenax Wedd. (5)

Pilea Lindl. (28)

Pouzolzia Gaud. (7)

Rousselia Gaudich. (1)

Urera Gaud. (8)

Urtica L. (10)

\section{Familia Valerianaceae}

Plectritis DC. (1)

Valeriana L. (38)

\section{Familia Verbenaceae}

Aegiphila Jacq. (13)

Aloysia A.Juss. (9)

Avicennia L. (2)

Bouchea Cham. (8)

Burroughsia Moldenke (1)

Callicarpa L. (3)

Citharexylum L. (36)

Clerodendrum L. (4)

Cornutia L. (2)

Duranta L. (2)

Glandularia J.F.Gmel. (17)
Lantana L. (20)

Lippia L. (31)

Petrea L. (1)

Phryma L. (1)

Phyla Lour. (2)

Priva Adans. (5)

Rehdera Moldenke (2)

Stachytarpheta Vahl (14)

Tamonea Aubl. (3)

Tectona L.f. (1)

Verbena L. (30)

Vitex L. (8)

*Xolocotzia Miranda (1)

\section{Familia Violaceae}

Corynostylis Mart. \& Zucc. (1)

Hybanthus Jacq. (16)

Ionidium Vent. (1)

Orthion Standl. \& Steyerm. (5)

Rinorea Aubl. (6)

Viola L. (29)

\section{Familia Viscaceae}

Arceuthobium M.Bieb. (19)

Dendrophthora Eichler (5)

Phoradendron Nutt. (78)

\section{Familia Vitaceae}

Ampelocissus Planch. (2)

Ampelopsis Michx. (3)

Cissus L. (17)

Parthenocissus Planch. (2)

Vitis L. (14)

Familia Vochysiaceae

Vochysia Aubl. (2)

Familia Winteraceae

Drimys J.R.Forst. (1)

Familia Zygophyllaceae

Fagonia L. (9)

Guaiacum L. (4)

Kallstroemia Scop. (13)

Larrea Cav. (1)

*Morkillia Rose \& Painter (2)

Peganum L. (1)

Porlieria Ruiz \& Pav. (1)

* Sericodes A.Gray (1)

*Viscainoa Greene (2) 